\title{
Handover Based IMS Registration Scheme for Next Generation Mobile Networks
}

\author{
Shireen Tahira, ${ }^{1}$ Muhammad Sher, ${ }^{1}$ Ata Ullah, ${ }^{2}$ \\ Muhammad Imran, ${ }^{3}$ and Athanasios V. Vasilakos ${ }^{4}$ \\ ${ }^{1}$ Department of Computer Science and Software Engineering, International Islamic University, Islamabad 44000, Pakistan \\ ${ }^{2}$ Department of Computer Science, National University of Modern Languages, Islamabad 44000, Pakistan \\ ${ }^{3}$ College of Computer and Information Sciences, King Saud University, Riyadh, Saudi Arabia \\ ${ }^{4}$ Department of Computer Science, Electrical and Space Engineering, Luleå University of Technology, 97187 Luleå, Sweden
}

Correspondence should be addressed to Shireen Tahira; shireentahira381@gmail.com

Received 27 May 2017; Revised 2 August 2017; Accepted 7 August 2017; Published 27 September 2017

Academic Editor: Syed Hassan Ahmed

Copyright (C) 2017 Shireen Tahira et al. This is an open access article distributed under the Creative Commons Attribution License, which permits unrestricted use, distribution, and reproduction in any medium, provided the original work is properly cited.

\begin{abstract}
Next generation mobile networks aim to provide faster speed and more capacity along with energy efficiency to support video streaming and massive data sharing in social and communication networks. In these networks, user equipment has to register with IP Multimedia Subsystem (IMS) which promises quality of service to the mobile users that frequently move across different access networks. After each handover caused due to mobility, IMS provides IPSec Security Association establishment and authentication phases. The main issue is that unnecessary reregistration after every handover results in latency and communication overhead. To tackle these issues, this paper presents a lightweight Fast IMS Mobility (FIM) registration scheme that avoids unnecessary conventional registration phases such as security associations, authentication, and authorization. FIM maintains a flag to avoid deregistration and sends a subsequent message to provide necessary parameters to IMS servers after mobility. It also handles the change of IP address for user equipment and transferring the security associations from old to new servers. We have validated the performance of FIM by developing a testbed consisting of IMS servers and user equipment. The experimental results demonstrate the performance supremacy of FIM. It reduces media disruption time, number of messages, and packet loss up to $67 \%, 100 \%$, and $61 \%$, respectively, as compared to preliminaries.
\end{abstract}

\section{Introduction}

Next generation mobile networks (NGMNs) provide faster speed and wide capacity for outdoor and indoor multimedia based applications in affordable rates for supporting large set of customers. It can benefit this era where social networks are sharing huge data and video transfer across the world. NGMN consists of two layers [1] including transport layer and service control layer where the former manages the connectivity and transmission using WiMAX, UMTS, and LTE and the latter utilizes IMS servers for registration, integration, charging, and policy enforcement as per 3rd Generation Partnership Project (3GPP). User equipment (UE) connects through transport layer and then registers with IP Multimedia Subsystem (IMS) using service control layer in most access networks such as LTE/LTE-A. UE is mostly connected to the Internet and can establish audio/video calls anywhere and anytime. During traveling, UE can move out of a current network range and may join another neighboring network by performing handover to support uninterruptible call sessions. To provide seamless vertical handover across different heterogeneous NGMNs, various solutions using IMS were proposed in the literature [2-5].

During vertical handover, UE has to deregister with the old network and reregister with the new in order to continue uninterrupted service, which also requires obtaining a new IP address from the latter and reregistering with IMS. Moreover, IPSec Security Associations (SAs) have to be established again between UE and Proxy-Call Session Control Function (P-CSCF) to secure the mutual communications. During handover scenarios, UE changes its P-CSCF which is the first entry point for reregistration at IMS as illustrated in Figure 1. 


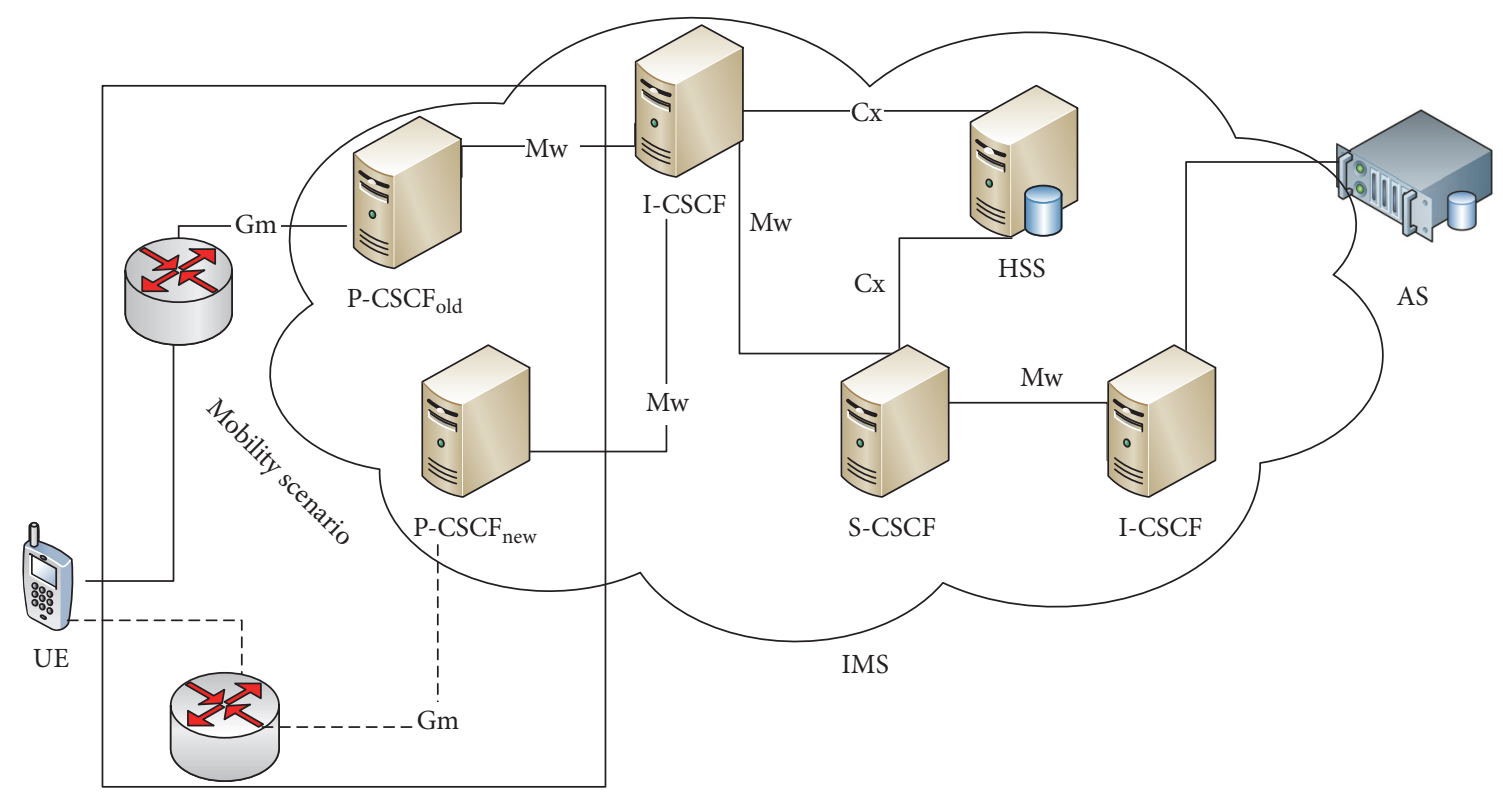

Mobility occurs when UE connects to new AR and new P-CSCF

FIGURE 1: Reregistration at IMS in handover scenario.

UE exchanges messages over $G m$ interface to initiate registration process with P-CSCF that further contacts InterrogatingCall Session Control Function (I-CSCF) by using $M w$ interface. I-CSCF is responsible to get the address of Serving-Call Session Control Function (S-CSCF) that is to be assigned to the UE. I-CSCF retrieves the address using $C x$ interface to contact Home Subscriber Server (HSS) that maintains subscription related information of UE in a database. Application Server (AS) hosts and executes the services offered by IMS. UE has to go through this phase each time it connects to IMS that involves four messages from UE to IMS. It increases latency and disruption time that affects QoS [6], increases number of messages [7], and causes more packet loss [8].

Some of the existing reregistration schemes proposed in the literature $[7,9,10]$ strived to reduce the number of messages and media disruption time (MDT), that is, time during which service is unavailable due to handover. Most of the schemes address delay in MDT by suggesting either integrating new protocols $[2,4,5,9-12]$ or context transfer from old servers to the new $[5,7]$. Preprocessing is suggested by [11-13] in order to save time. The issue of changing the IP address of UE during handover is also addressed in [9] but not within Session Initiation Protocol (SIP) capabilities. SIP [14] is the main protocol of IMS for creating, modifying, and terminating sessions between UE and Corresponding Node $(\mathrm{CN})$. Minimizing number of messages is addressed in [7, 9]. Mobile IPv6 (MIPv6) [15] and Fast MIPv6 (FMIPv6) in IMS solve mobility and latency problems. Media Independent Handover (MIH) [16] is also exploited to get mobility information of target network for preregistration in many schemes in order to reduce latency of layer 2 mobility. But none of the above methods provided the solution for authentication and authorization of entities needed in IMS registration after handover. In 3 GPP specification, a new entity named service centralization and continuity application server is introduced for service continuity by maintaining long-term session between UE and $\mathrm{CN}$ on a new IP address. It ensures service continuity but it causes delay during registration phase.

The main problem during registration is that it undergoes latency in MDT, loss of packets, and more number of commands/messages. From literature, we have identified that there is a need to optimize the registration phase with less communication overhead. It should also cater transfer of IPSec SAs to provide authentication and authorization. The scheme should be generic that can be used with any NGMNs handovers between either LTE and WiMAX or LTE and 5G. Until now cross-layer schemes are proposed that reduce the registration delay but only by utilizing either layer 2 or layer 3 protocols. This paper presents a Fast IMS Mobility (FIM) scheme that maintains a flag to ensure that no deregistration in IMS occurs if flag is enabled during handover. During handover, UE does not intentionally terminate from AN and keeps a session with $\mathrm{CN}$ to get registered in IMS again. We have recommended that, after layer 3 handover and discovery of new P-CSCF, UE should transmit a new subsequent request for handover to S-CSCF for replacing old IP address of UE with new one. It also initiates transferring IPSec SAs to new P-CSCF. In this way, four messages of REGISTER request are reduced to two messages. Similarly it reduces number of commands by eliminating the need of network initiated deregistration in order to reduce communication overhead. We have set up a testbed for IMS along with supported servers and UE to implement and validate the handover process. Results prove the dominance of FIM as compared to IMS handover process and MIP based handover schemes in terms of minimizing the MDT, packet loss, and number of commands.

The rest of the paper is organized as follows. Section 2 describes the system model and related schemes for handover 


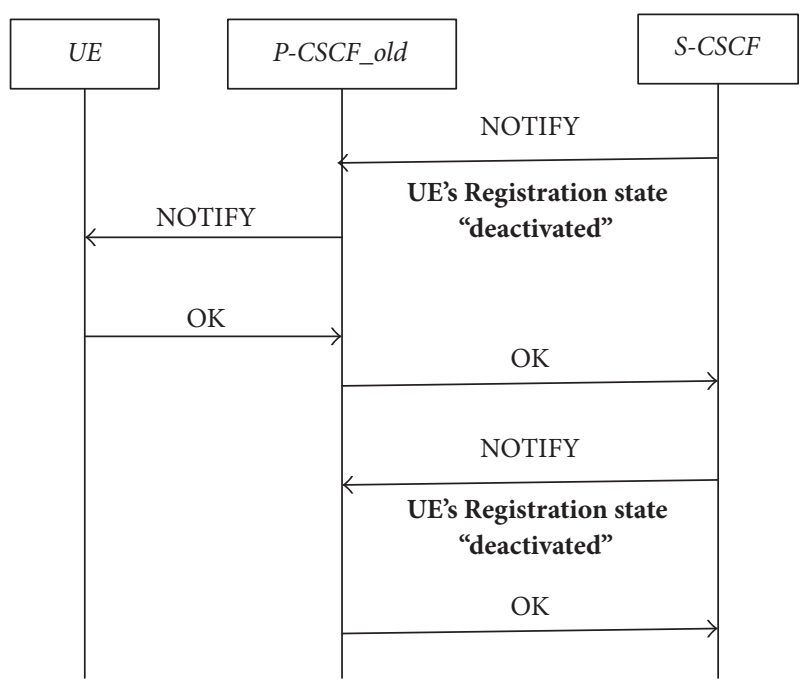

FIgURE 2: Network initiated deregistration in IMS.

in NGMN using IMS are discussed in Section 3. The proposed scheme is described in Section 4. Section 5 elucidates testbed implementation, results, and analysis. Finally, our work is concluded in Section 6.

\section{System Model}

We consider an IMS model where the main entities are CSCF servers, its database, and application server [17]. Figure 2 illustrates the flow of steps during network initiated deregistration in IMS during handover. After disconnection of UE from AN, the S-CSCF sends a NOTIFY message to P-CSCF_Old that forwards it to UE to inform it about deactivation of its registration. UE acknowledges it by sending OK response. S-CSCF also notifies old P-CSCF about deactivation of UE and old P-CSCF responds by OK message.

On connecting to a new AN, UE gets registered to IMS via new P-CSCF. Initially, a REGISTER request is sent to new P-CSCF who forwards it to I-CSCF that shares user authentication request (UAR) and answers UAA with HSS. After that I-CSCF transmits the REGISTER request to S-CSCF that exchanges message authentication request (MAR) and answers (MAA) with HSS and then replies with the 401 unauthorized message. Upon receiving 401, UE again transmits a REGISTER message to S-CSCF through P-CSCF and I-CSCF. $S$-CSCF exchanges server authentication request (SAR) and answers SAA with HSS and then sends OK message as shown in Figure 3. IPSec SAs are also established between UE and PCSCF to ensure that these messages are exchanged between authorized UE and servers. During registration, the SIP headers including Via, Route, To, Contact, Path, and ServiceRoute are also used. Every time UE gets connected to new AN, the whole phase of registration is carried out that increases the delay in MDT and number of commands. Delay in MDT causes degraded QoS because of interruption in data flow and loss of packets. We assume that an intrusion detection system is functional to guard against security breaches and failure of messages by adversaries. It is also assumed that neighboring

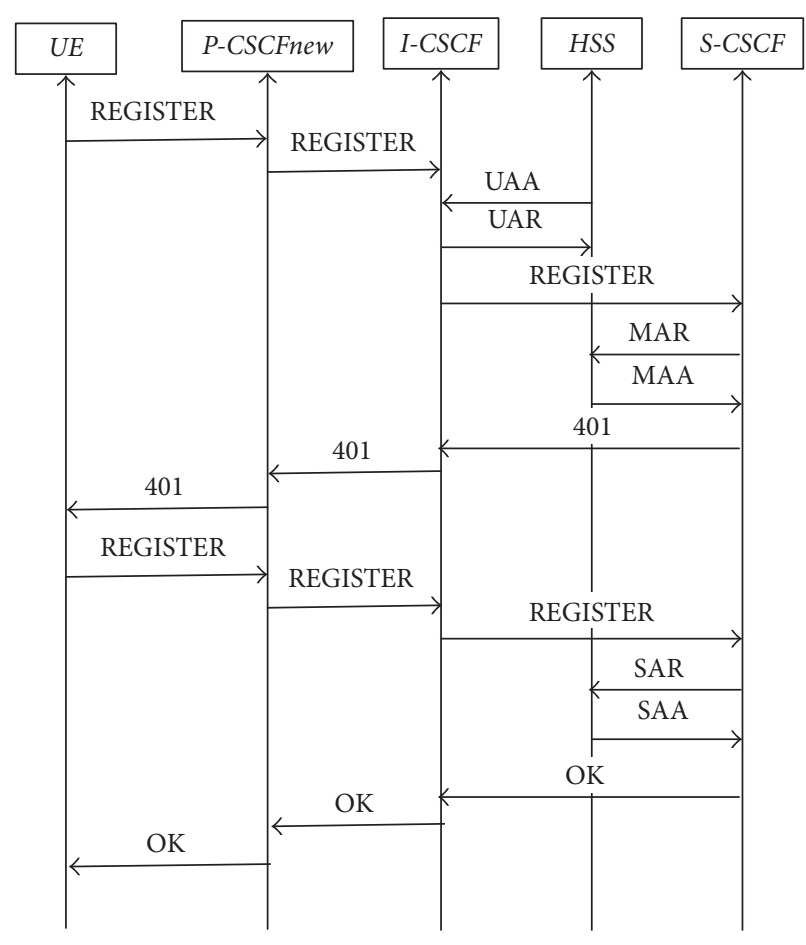

FIGURE 3: UE's registration at IMS.

AN is trustworthy to transfer session credential during handover and change of IP address is protected from IP spoofing.

\section{Related Work}

In this section, we discuss different schemes for handover scenarios and related functionalities by using IMS. Related schemes are discussed under different categories including handover schemes with less MDT where the target is to reduce the MDT during the handover process. It is further subdivided into new protocol inclusion based schemes, context transfer, preprocessing, simultaneous processing, and minimizing number of commands based schemes. In addition to these, change in IP address based schemes and packet loss evaluation based schemes are also discussed in the following section.

3.1. Handover Schemes with Less MDT. In literature, we have studied many techniques that are proposed to reduce MDT. We have discussed related schemes under different categories including integration of new protocols, context transfer, preprocessing, and simultaneous processing.

3.1.1. New Protocol Inclusion in IMS Based Schemes. These schemes recommend including new protocols in IMS for reducing MDT. FMIPv6 is developed for reducing layer 3 delay [18] that is recommended to be integrated with IMS to reduce handover delay [10,11]. Figure 4 shows the MIP based mobility solution for IMS handover where two messages are exchanged between UE and old Access Router (ARold) for router solicitation/advertisement. Two messages are exchanged between UE and Home Agent (HA) for Binding 


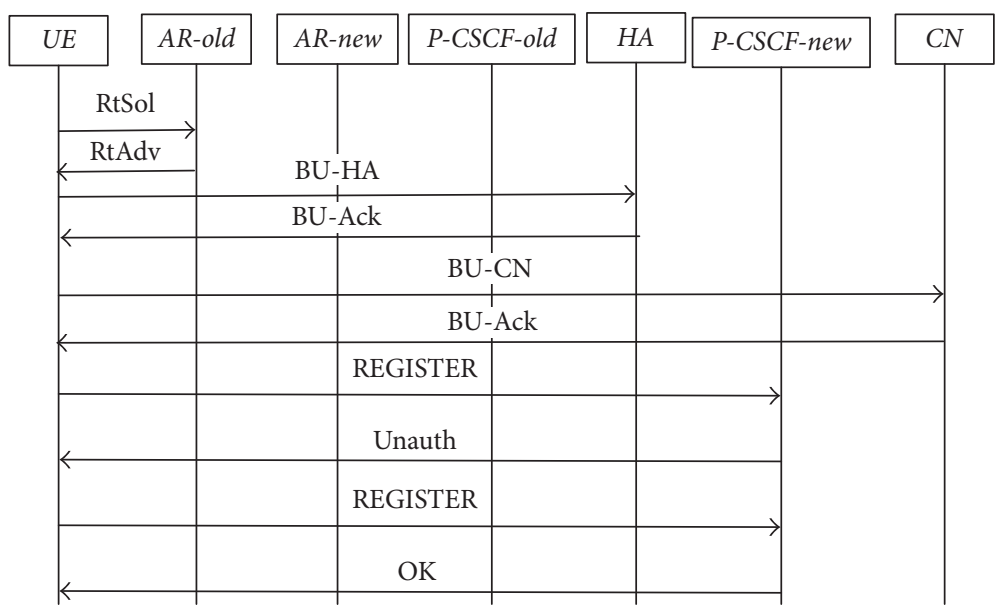

FIGURE 4: MIP-IMS handover.

Update (BU) and BU Acknowledgment (BU-Ack). Similarly two messages are exchanged between $\mathrm{UE}$ and $\mathrm{CN}$ for $\mathrm{BU}$ and BU-Ack. Then standard registration phase is carried out via new P-CSCF. Thanh et al. [19] have presented a solution to integrate mSCTP with IMS with a feature of multihome. When handover occurs, UE establishes a new mSCTP connection with new network while the old connection with previous network is still active. A new SWITCH SIP method is proposed in order to quickly switch between these two networks. Once the second mSCTP connection is established, switching between two networks is done with less latency. But in this way application layer latency caused by handover is not minimized and the integration issues of MIPv6 in IMS are inherited in FMIPv6 as well [6].

A cross-layer architecture has been proposed for handover from LTE to WiMAX by integrating MIP and SIP protocols [2]. It uses evolved packet core as the core network and IMS to provide multimedia services and manage sessions. The delay $(D)$ for handover from LTE to WiMAX is calculated using (1), where $D_{\text {MIP }}$ is calculated using (2) by calculating the delay time of agent solicitation/advertisement for the Base Station (BS) of WiMAX and registration request and reply with HA to identify the new P-CSCF and S-CSCF. The time $T_{\text {UEtoCN }}$ is calculated for BU and BU-Ack messages that are exchanged between $\mathrm{UE}$ and $\mathrm{CN}$.

$$
\begin{aligned}
D & =D_{\mathrm{MIP}}+D_{\mathrm{SIP}}+D_{\mathrm{HSS}} \\
D_{\mathrm{MIP}} & =2 T_{\mathrm{UEtoBS}}+2 T_{\text {UEtoHA }}+2 T_{\text {UEtoCN }} .
\end{aligned}
$$

Equation (3) explores the calculation of $D_{\text {SIP }}$ for non-crosslayer architecture that involves the delay time taken for the registration request and response messages between UE and S-CSCF. It also involves delay time for 3 Re-INVITE messages between UE and CN, that is, REINVITE, OK, and ACKNOWLEDGMENT. Equation (4) shows $D_{\text {SIP_CL }}$ for cross-layer scenario.

$$
\begin{aligned}
D_{\text {SIP }} & =2 T_{\text {UEtoS }}+3 T_{\text {UEtoCN }} \\
D_{\text {SIP_CL }} & =2 T_{\text {HAtoS }}+3 T_{\text {UEtoCN }}
\end{aligned}
$$

In this case, $D_{\mathrm{HSS}}$ is the delay consumed by S-CSCF to update HSS about new location of UE. In this scheme registration of UE with S-CSCF is shown in 2 messages but it does not explain the authentication, establishment of SAs with new P$\mathrm{CSCF}$, and integrity protection issues.

3.1.2. Context Transfer Based Schemes. Techniques proposed in $[7,9]$ transfer the context from old P-CSCF to new PCSCF for reducing number of messages for registration and session establishment that leads to reduction in MDT. It also contains the information of the user and state of session that was being used before the UE changes its network. Thus it reduces number of messages and handover latency. Farahbakhsh and Movahhedinia [10] proposed two schemes for supporting seamless handover and two schemes for QoS parameter negotiation. These schemes are proposed to reduce latency by transferring the context from old servers to new servers. These techniques are either predictive (user knows in advance to which network it has to move) or reactive. These techniques use FMIPv6 to remove latency of handover. Equation (5) shows its predictive scheme where four messages are exchanged between UE and old AR ( $\left.4 T_{\text {UEtoARo }}\right)$. One message is sent to old P-CSCF from UE ( $\left.T_{\text {UEtoPold }}\right)$. Two messages are exchanged between old AR and new AR ( $\left.2 T_{\text {ARotoARn }}\right)$. One message is sent from UE to new AR $\left(T_{\text {UEtoARn }}\right)$. Two messages are exchanged between UE and HA $\left(2 T_{\mathrm{HA}}\right)$. Four messages are exchanged between UE and $\mathrm{CN}\left(4 T_{\text {UEtoCN }}\right)$. Two messages are exchanged between UE and new P-CSCF ( $\left.2 T_{\text {UEtoPnew }}\right)$. Equation (6) shows $\mathrm{MDT}_{\mathrm{RM}}$ for reactive mode where $T_{\text {Poldtos }}$ is the message between old P-CSCF and S-CSCF.

$$
\begin{aligned}
\text { MDT }= & 4 T_{\text {UEtoARo }}+T_{\text {UEtoPold }}+2 T_{\text {ARotoARn }} \\
& +T_{\text {UEtoARn }}+2 T_{\text {HA }}+4 T_{\text {UEtoCN }} \\
& +2 T_{\text {UEtoPnew }} \\
\text { MDT }_{\text {RM }}= & 2 T_{\text {UEtoARo }}+3 T_{\text {UEtoPnew }}+T_{\text {UEtoARn }} \\
& +2 T_{\text {ARotoARn }}+3 T_{\text {PoldtoPnew }}+2 T_{\text {PoldtoS }} \\
& +2 T_{\text {PoldtoS }}+2 T_{\text {HA }}+4 T_{\text {UEtoCN }} .
\end{aligned}
$$


3.1.3. Preprocessing Based Schemes. Few researchers introduced the approaches to do some kind of preprocessing before doing any handover to a new network. UE connects to a new network and then it rejects it for the reason that it does not provide required QoS and goes for the selection of a new network repeatedly till it finds the best suitable network. This process also adds up in MDT due to handover to new networks in the hunt of best network. Ito et al. [11] have proposed that MDT can be minimized by starting the service continuity procedure as soon as UE gets an IP address proactively due to FMIPv6. Buffering should be performed to minimize packet loss. If the network is not suitable one, then UE does not register in IMS with this network; rather UE searches for next available network. Nazari et al. [12] proposed a solution to minimize handover delay by eliminating the delay of registration in target network and IMS. With the help of MIH protocol [20], handover server enables the UE to get mobility information and then it preregisters with new network and IMS without being in coverage area. For preregistration in core network, authentication process should be done. For authentication process EAP (Extensible Authentication Protocol) is carried out. In this method, a handover between WiMAX and LTE is illustrated. For a handover to LTE, the security key used in the target BS cannot be constructed without knowing the BS's identity. Similarly in case of handover to WiMAX, master session key is generated and sent to target network but security key cannot be generated until identity of the BS is known. So the preregistration in the BS $\left(D_{\mathrm{B}-\mathrm{PR}}\right)$ is done when UE enters in the coverage area. Therefore the delay of handover preparation is equal to time of registration in the BS only. MDT is calculated as Delay $=D_{(\mathrm{B}-\mathrm{PR})}$, where $D_{\mathrm{B}-\mathrm{PR}}$ is the preregistration in the BS.

Yang and Chen have proposed a QoS reservation model [21] that reserves resources in advance at neighboring IMS networks and manages mobility by using SIP multicast [22]. During handover between LTE and WiMAX, a cross-layer architecture $[4,5]$ recommends sending prior Re-INVITE to new AN while it is connected to old AN. During the reregistration phase, two messages are exchanged that contain IPSec SA parameters and UE's new IP address. The authors claimed that the total MDT in this scheme is only the IMS delay because switching to underlying link by UE is parallel to session setup. Moreover, the prior duplicate address detection [23] delay at layer 3 is also removed by using MIH services. For vertical handover between $3 \mathrm{G}$ and $\mathrm{WiFi}$, many schemes are proposed to reduce delay by preregistration and pre-ReINVITE [24]. Moon proposed an IPSec SA establishment method [3] in such schemes. It adds new SIP headers to resolve the IP address mismatch problem for IPSec SAs by indicating the destination IP address of UE in its SIP messages. Thus no detail is mentioned about how layer 3 mechanism reduces the overall MDT.

3.1.4. Simultaneous Processing Based Scheme. Another approach to reduce handover delay is to keep processing in old network while working on new network. Bellavista et al. have proposed a framework [25] where a module predicts the network on which UE is going to handover its connection. It keeps the flow on old network and also starts signaling on new network. Another module is to handle QoS issue on new network. In all these existing schemes, UE still has to get registered in IMS with new IP address and the registration/authentication process also runs once again that adds delay to MDT.

3.1.5. Minimizing Number of Commands Based Schemes. Larsen et al. proposed a scheme in order to reduce number of SIP messages for registration and invite (for session) in order to reduce MDT [7]. It proposed reusing the information about user and session states in CSCF servers. This technique claims to reduce number of messages from 15 to 4 . It transfers the context from old P-CSCF to new P-CSCF, which contains the information of the user and state of session that was going on before user changed the network. It does not explain the methods of secure transfer of IPSec SAs to new P-CSCF, authentication, and authorization of UE.

3.2. Change of IP Address Based Schemes. Different schemes recommend integrating Mobile IP [9] in IMS as SIP does not provide a solution to change IP address of UE after handover. MIPv6 [15] is a layer 3 protocol for providing mobility solutions and reduces layer 3 mobility delay. It hides mobility of UE from upper layers. On changing AN, UE gains a Care of Address (CoA) that is saved in another entity Home Agent (HA). Communication is done by addressing Home Address (HoA) of UE. Message destined to UE goes to HA that finds the CoA of UE and forwards the messages to UE. Upper layers are unaware of the mobility of UE. MIP has integration issues that are discussed in [6].

3.3. Packet Loss Evaluation Based Schemes. Bagubali et al. [8] analyzed and evaluated the IMS based integration architecture proposed in [2] for WiMax/LTE handover. Crosslayer architecture is better than simple layered architecture by evaluating packet losses and other parameters [8]. Equation (7) is used by [26] to measure packet loss where $\mathrm{Ti}_{\mathrm{ad}}$ is agent advertisement signal, $G$ is downlink packet transmission rate, $D$ is handoff delay, and $\mathrm{Nm}$ is the number of handovers during a session.

$$
\text { Packet Loss }=\left[\left(\frac{1}{\left(2 * \mathrm{Ti}_{\mathrm{ad}}\right)}\right)+D\right] * G * \mathrm{Nm} \text {. }
$$

\section{Fast IMS Mobility (FIM) Scheme}

This section discusses our proposed scheme that handles the handover and registration process when users are moving in a region. During mobility scenarios, UE needs to be transferred from one AN to the other without interrupting its session. Authentication and authorization of UE are also needed after handover so these are also catered in our scheme. Our scheme also gives a mechanism to transfer IPSec SAs from old PCSCF to new P-CSCF in a secure manner. FIM scheme benefits all NGMN including $4 \mathrm{G}$ and $5 \mathrm{G}$ because it gives the solutions on application layer. The notations used in FIM are listed in the Notations.

In our scheme, the communication begins when UE transmits a proposed subsequent HANDOVER request to old 


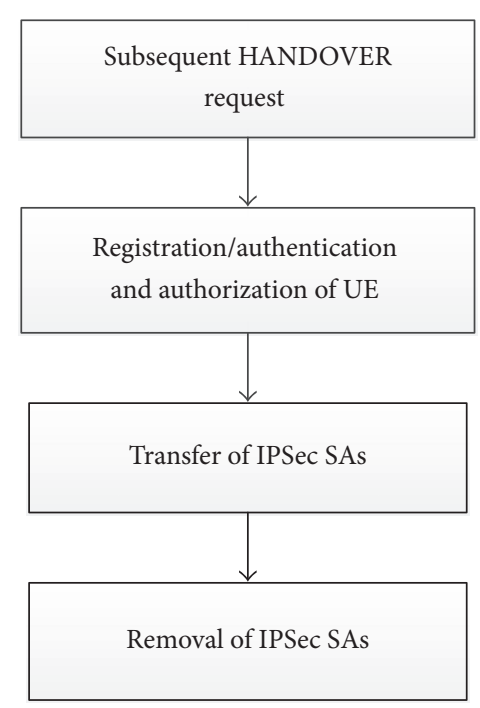

FIGURE 5: Phases of FIM scheme during handover.

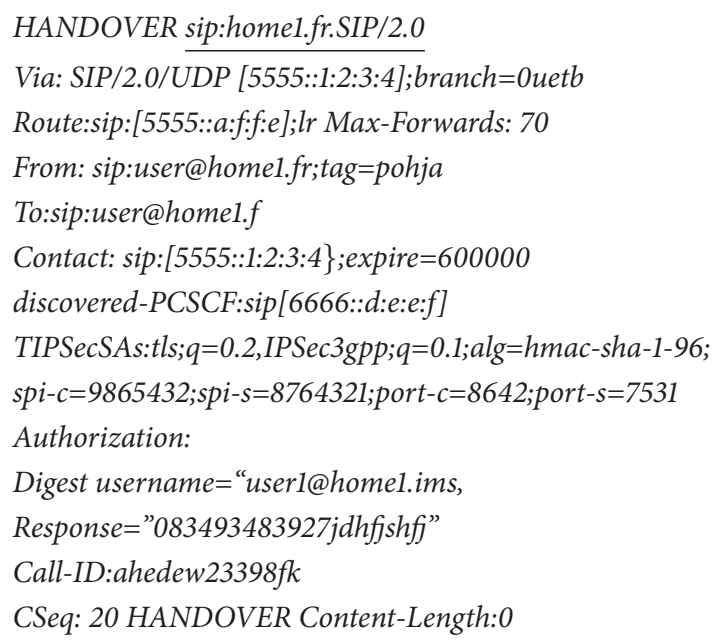

Box 1: Subsequent HANDOVER request message.

P-CSCF. HANDOVER request is sent instead of REGISTER request on the basis of status of "sessionContinued" flag in case it is enabled. It ensures that the UE and $\mathrm{CN}$ have not cancelled the session between them and the UE is disconnected from $\mathrm{AN}$ for a short period that could be the handover purpose. After that, UE's registration/authentication process begins at S-CSCF to proceed with the connectivity of new P-CSCF along with credentials. In the next phase, IPSec SAs are forwarded by the S-CSCF to new P-CSCF that exchanges the confirmation messages for the running call session. Finally, the old P-CSCF removes the IPSec SAs from itself as illustrated in four phases in Figure 5.

In the first phase, UE transmits a subsequent HANDOVER request with its new IP address in Contact and IP address of P-CSCF in discovered-PCSCF header as shown in Box 1. If the flag is not set, then REGISTER request will be sent to old-P-CSCF as illustrated in Pseudocode 1. UE's

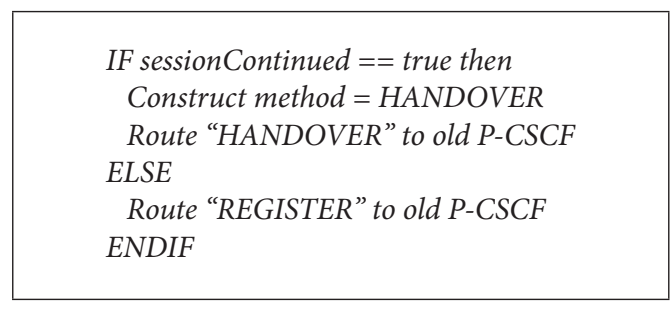

Pseudocode 1: Session handling at UE.

disconnection from an AN leads to deregistration from IMS. Our scheme avoids deregistration due to sessionContinued flag. In this way, all the related data of UE's registration is not deleted from the IMS servers and UE. After layer 3 mobility, UE discovers a P-CSCF that is either new one or the same one at previous location. FIM sends HANDOVER request to S-CSCF via old P-CSCF instead of starting registration. The HANDOVER request utilizes a routing header, that is, Service-Route. In this way, P-CSCF does not need to contact I-CSCF for S-CSCF's address. So the I-CSCF does not contact HSS that results in no $C x$ commands for our scheme.

In the second phase, $\mathrm{UE}$ connects to a new $\mathrm{AN}$ and verifies that if the status of sessionContinued flag is enabled, then, instead of sending REGISTER request to IMS, UE constructs HANDOVER request. UE integrates its new IP address in Via and Contact headers. UE places IP address of new P-CSCF in a new proposed header discovered-PCSCF in HANDOVER message. UE forwards this HANDOVER request to old PCSCF that forwards it to S-CSCF where new values from Contact and discovered-PCSCF headers are saved. IP address of new P-CSCF is copied to path. Now S-CSCF is able to forward request destined for UE towards new P-CSCF. In this way, UE gets registered in IMS. UE and HSS keep a shared secret and sequence number. In UE, it is saved in ISIM application on universal integrated circuit card (UICC). In the initial REGISTER request of standard IMS scheme, S-CSCF downloads authentication vectors (AVs) for UE. AVs contain random challenge (RAND) and expected response (XRES) along with integrity key (IK), cipher key (CK), and authentication token (AUTN). S-CSCF sends these AVs to UE in initial request's response, that is, 401. UE calculates RES with the help of shared secret and RAND. This RES is like a password [27] and used for authentication. In our proposed scheme, due to no deregistration, RES and XRES are not lost and used for authentication during HANDOVER phase. When S-CSCF receives HANDOVER, it compares the received RES with XRES and then saves the new IP address of UE and IP address of P-CSCF as illustrated in Pseudocode 2. In other scenarios, when the method is not HANDOVER, then complete registration process is performed including user RES verification in the reply of "Unauthorized" message. During registration, UE and P-CSCF establish IPSec SAs that ensures integrity protection/authorization. Our scheme sends HANDOVER request through old P-CSCF where IPSec SAs are saved in a header. By sending the request through old P-CSCF, the integrity of messages is ensured because UE and old P-CSCF have already established IPSec SAs between them. Thus it guarantees that messages are exchanged between 


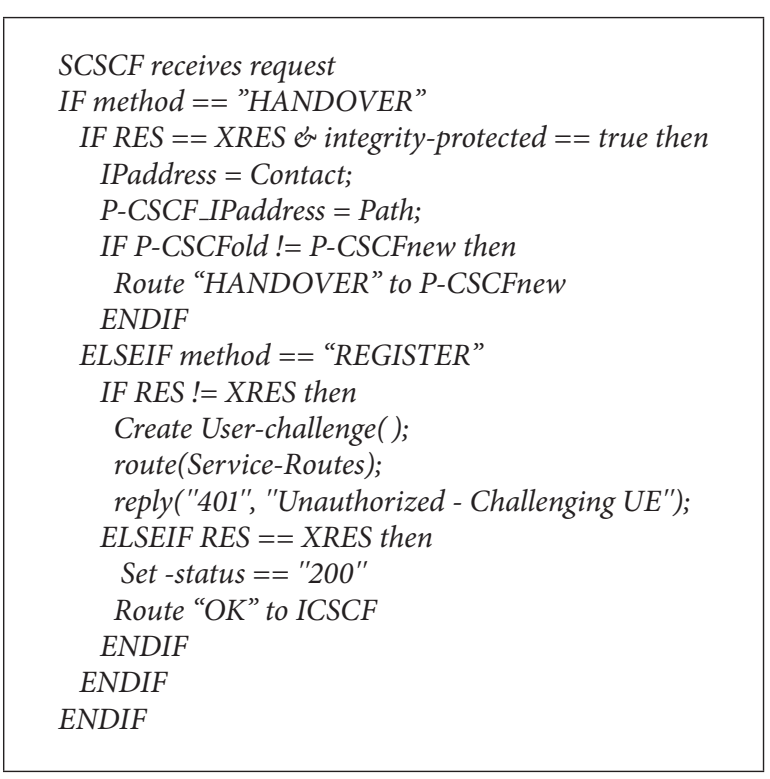

Pseudocode 2: Authentication at S-CSCF.

trusted entities. In this way, our scheme handles registration, authentication, and authorization in two messages.

In the third phase, the old P-CSCF receives message from UE and compares that if the received request is HANDOVER, then add IPSec SAs to HANDOVER message in TIPSecSAs header. It adds the IP address of new P-CSCF to path header and set Integrity-Protected to yes in HANDOVER request. Old P-CSCF does not send IPSec SAs to new P-CSCF directly because there is no IPSec SAs tunnel between these two entities. In our scheme, IPSec SAs are transferred from old P-CSCF to S-CSCF. S-CSCF first compares RES with XRES and then transfers the received IPSec SAs to new P-CSCF. Thus the transfer of IPSec SAs from old P-CSCF to new PCSCF is done in a secure manner with authenticity. This is only done when the newly discovered P-CSCF is not the old one (before handover) because the transfer is only needed in that case. In this way, no new protocol is used to transfer IPSec SAs. No new commands are used for the transfer of IPSec SAs as it is done within the handover phase proposed by our scheme. In the fourth phase, S-CSCF transmits "OK" message to old P-CSCF. As in our scheme, there is no deregistration, so in order to delete IPSec SAs from old P-CSCF, the entity removes IPSec SAs from itself after sending "OK" message to UE as shown in Pseudocode 3. It is only needed when newly discovered P-CSCF is the new one. The registration after handover is presented in a visual manner in Figure 6 by sequentially exploring the above four phases.

In this way, we have reduced MDT between two entities after handover from one AN to another. MDT is confined by reducing delay and cost of the registration phase in IMS. Similarly we proposed reducing number of $G m, M w$, and $C x$ commands. Our proposed scheme handles authorization of UE and transfers IPSec SAs during registration phase. It handles all this with low latency in MDT as given in (8), where $E=\{\mathrm{UE}, \mathrm{PCSCF}, \mathrm{ICSCF}, \mathrm{HSS}, \mathrm{SCSCF}\}$ is the set of entities, $x$ is the number of messages between two entities, and $T\left(E_{i}, E_{j}\right)$ is delay time taken by the message between entities $E_{i}$ and $E_{j}$, where $i=\{1,2,3,4\}$ and $j=i+1$.

$$
\text { minimize MDT }=\sum x \cdot T\left(E_{i}, E_{j}\right)
$$

For minimizing number of commands, if we consider $S=$ \{PCSCF, ICSCF, SCSCF $\}$ is the set of IMS servers and $H=$ $\{$ HSS $\}$ is the set of database. Here $y$ is the number of $M w$ commands, $i=\{1,2\}$. Then number of $M w$ commands is needed to be minimized as given in equation (9). It calculates sum of $M w$ commands needed from P-CSCF to I-CSCF and from P-CSCF to S-CSCF. Equation (10) calculates sum of $C x$ commands needed from I-CSCF to HSS and from I-CSCF to HSS where $z$ is the number of $C x$ commands.

$$
\begin{aligned}
M w \text { commands } & =\sum y \cdot\left(S_{i}, S_{i+1}\right) \\
C x \text { commands } & =\sum z \cdot\left(S_{i+1}, H\right) .
\end{aligned}
$$

$U=\{\mathrm{UE}\}$ and $k$ is the number of $G m$ commands; then the minimization of $G m$ commands is given in (11) that calculates the sum of $\mathrm{Gm}$ commands needed from P-CSCF to UE.

$$
\text { Gm commands }=\sum k \cdot\left(S_{i}, U\right) .
$$

\section{Results and Analysis}

The proposed FIM scheme is analyzed by developing a testbed using Open IMS core [28] to set up IMS entities including HSS, P-CSCFs, I-CSCF, and S-CSCF as illustrated in Figure 7. It is connected through IP network with four ARs where each one has a router and 802.11 g WLAN Access Point (AP). UE and $\mathrm{CN}$ are android phones that are connected to AN through WLAN AP. There are four ANs that individually contain $\mathrm{SSID}_{1}$ to $\mathrm{SSID}_{4}$ where UE disconnects from $\mathrm{SSID}_{1}$ and reconnects to $\mathrm{SSID}_{2}$. UE and APs establish session and exchange real-time transport protocol packets encoded with G.711 codec at $20 \mathrm{~ms}$ interval. UE also monitors the signaling strength of APs, and when the exponential smoothing value of the strength $\left(S_{t}=a S_{t-1}+(1-a) a_{t}, 0 \leq a \leq 1\right)$ goes below the threshold value, then UE connects to new AP with strong signal strength, where $a_{t}$ is signaling strength of AP at time $t$ and $S_{t}$ is the result at time $t$ and $a=0.5$ in the experiment as in [11]. For analysis of results, MDT of FIM is compared with MIP-IMS [2, 7] and standard IMS [17] scheme. Delay is considered as in [10], and number of hops and number of variations are considered as in [8]. Evaluation parameters for testbed are listed in Table 1. Moreover, for MIP-IMS, a router is deployed that maps the HoA with CoA and acts as HA. We have performed a number of handovers that can be categorized in three scenarios including Same P-CSCF (SP), Other Far P-CSCF (OFP), and Other Near P-CSCF (ONP) as discussed below.

(a) SP: It is handover scenario where UE disconnects from $\mathrm{AN}$ and then connects to a new AN but it gets attached to IMS with the same P-CSCF after handover.

(b) OFP: After handover to new AN, UE connects to a new P-CSCF that is physically located farther than old P-CSCF. 


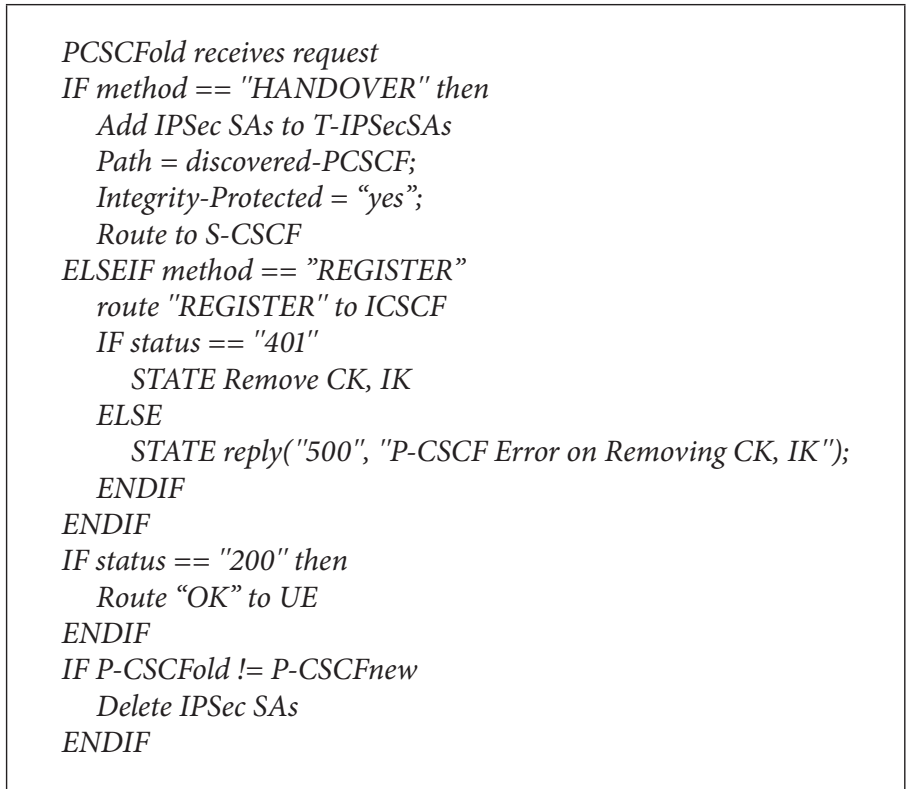

Pseudocode 3: IPSec SAs handling at old P-CSCF.

TABLE 1: Evaluation parameters for testbed.

\begin{tabular}{lc}
\hline & Testbed setup \\
Parameters & Values \\
\hline $\begin{array}{l}\text { Network servers } \\
\text { Servers' physical type } \\
\text { UEs' physical type }\end{array}$ & P-CSCF, I-CSCF, S-CSCF, HSS \\
Antenna type & Wired physical \\
& Wireless physical \\
Delay & Omni antenna \\
Number of hops & $0-90$ milliseconds \\
Number of handovers & $1-5$ \\
\end{tabular}

(c) ONP: UE connects to new P-CSCF where the distance of new P-CSCF is physically less than the old P-CSCF from UE.

5.1. Media Disruption Time. It is the time delay that occurred due to credential transfer during handover. In standard IMS, handover MDT can be calculated as given in (12), where $T_{\text {UEtoARn }}$ is the time taken for UE to connect to new AR and $T_{\text {UEtoP }}$ is time taken by UE to get itself registered at PCSCF. Time for REGISTER request to and from P-CSCF and $\mathrm{S}$-CSCF is represented as $T_{\mathrm{PtoS}}$. Time taken by REGISTER request to move between CSCF servers and HSS is denoted by $T_{\mathrm{CSCF}}$ toHSS and time for INVITE request for session establishment between $\mathrm{UE}$ and $\mathrm{CN}$ in SIP is represented as $T_{\text {UEtoCN }}$. Schemes $[2,4,5]$ use Re-INVITE request after handover where three messages are exchanged between UE and CN. In MIP based schemes and FIM scheme, Re-INVITE method is considered for correct comparison. In standard IMS method, there is no scheme for handover so INVITE method is used. In our experiments, we implemented MIP based IMS mobility scheme using HA to measure MDT as given in (13), where $T_{\mathrm{HA}}+T_{\mathrm{UEtoCN}}$ represents the time taken for sending Binding Update to CN and HA. Similarly, FIM scheme is implemented to measure MDT as given in (14)-(15).

$$
\begin{aligned}
& \mathrm{MDT}_{\mathrm{IMS}}=2 T_{\mathrm{UEtoARn}}+4 T_{\mathrm{UEtoP}}+4 T_{\mathrm{PtoS}} \\
& +6 T_{\text {CSCFtoHSS }}+8 T_{\text {UEtoCN }} \\
& \mathrm{MDT}_{\mathrm{MIP}}=2 T_{\text {UEtoARn }}+2 T_{\mathrm{HA}} \\
& +5 T_{\text {UEtoCN }}+4 T_{\text {UEtoP }}+4 T_{\text {Ptos }} \\
& +6 T_{\text {CSCFtoHSS }} \\
& \mathrm{MDT}_{\mathrm{SP}}=2 T_{\text {UEtoARn }}+T_{\text {UEtoPold }}+T_{\text {PoldtoS }} \\
& +3 T_{\text {UEtoCN }}+T_{\text {PnewtoUE }} \\
& \mathrm{MDT}_{\text {OFP_ONP }}=2 T_{\text {UEtoARn }}+T_{\text {UEtoPold }}+T_{\text {PoldtoS }} \\
& +2 T_{\text {StoPnew }}+3 T_{\text {UEtoCN }} \\
& +T_{\text {PnewtoUE }}
\end{aligned}
$$

To evaluate MDT, we set $T_{\text {UEtoRAN }}=10 \mathrm{~ms}$ as in $[29,30]$ and $T_{\text {UEtoARo }}=11 \mathrm{~ms}, T_{\text {ARotoARn }}=5 \mathrm{~ms}, T_{\text {UEtoPold }}=15 \mathrm{~ms}$, $T_{\text {PoldtoPnew }}=7 \mathrm{~ms}$, and $T_{\text {PoldtoS }}=10 \mathrm{~ms}$ as in [10]. We considered Internet delay as $100 \mathrm{~ms}$ and $T_{\mathrm{HA}}=116 \mathrm{~ms}, T_{\mathrm{UEtoCN}}$ $=128 \mathrm{~ms}$, and $T_{\text {HAtoCN }}=114 \mathrm{~ms}$ as in [10]. We assumed $T_{\text {UEtoARn }}=10 \mathrm{~ms}, T_{\text {PnewtoUE }}=16 \mathrm{~ms}$, and $T_{\text {StoPnew }}=12 \mathrm{~ms}$. MDT versus delay between UE and new AR is obtained for SP, OFP, and ONP scenarios. It can be seen that, for IMS standard scheme, MDT is increasing with a high rate. In Figure 8(a) for SP scenario, for a delay of $30 \mathrm{~ms}$, the MDT values are $338 \mathrm{~ms}$, $408 \mathrm{~ms}$, and $80 \mathrm{~ms}$ for IMS, MIP-IMS, and FIM, respectively, 


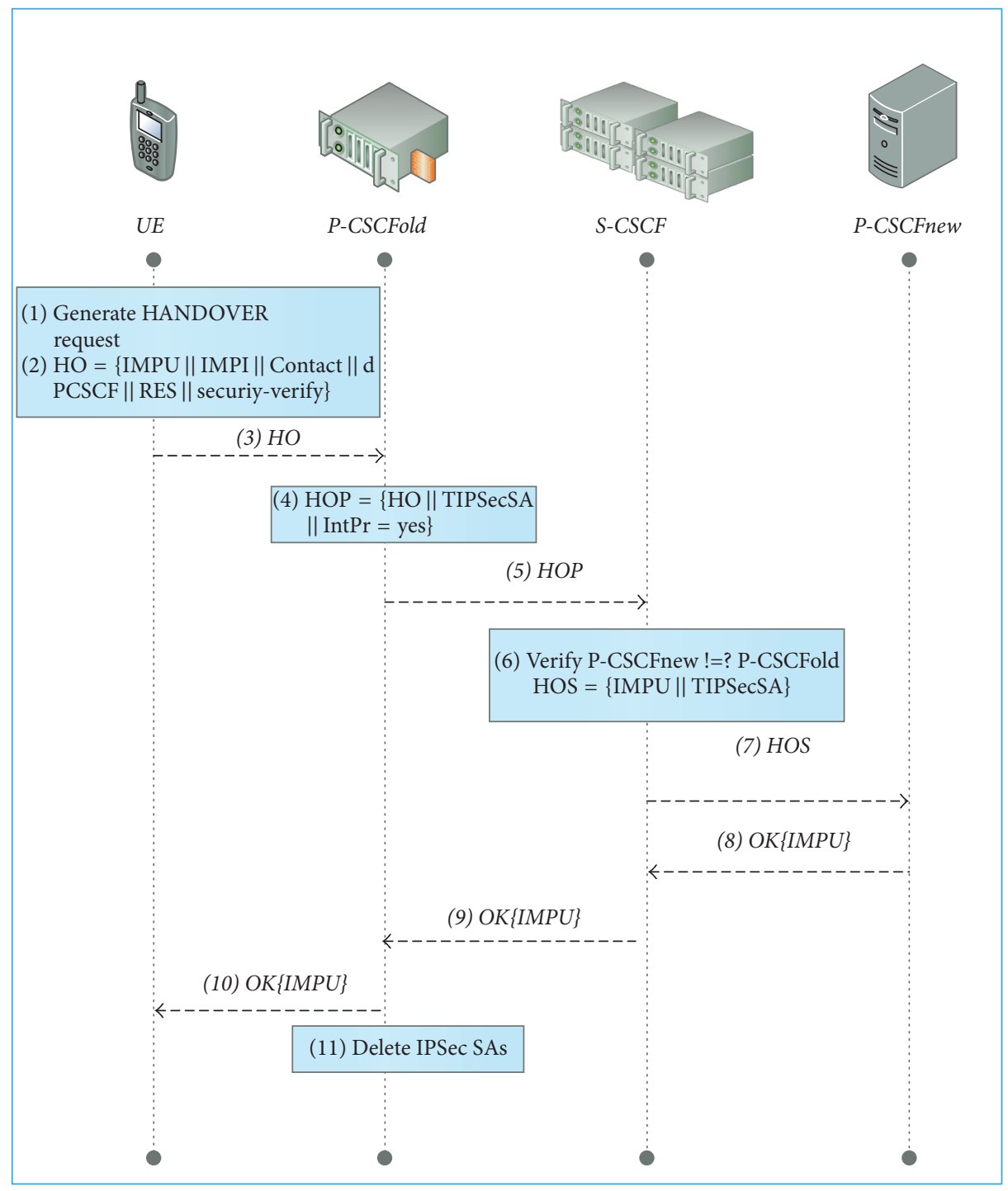

FIGURE 6: Proposed flow for FIM scheme.

where FIM requires the lowest MDT. It is also observed that when delay is increased three times, that is, $90 \mathrm{~ms}$, then MDT values are $458 \mathrm{~ms}, 528 \mathrm{~ms}$, and $122 \mathrm{~ms}$ for IMS, MIP-IMS, and FIM, respectively. In Figure 8(b) of OFP scenario, for a delay of $30 \mathrm{~ms}$, the MDT values are $338 \mathrm{~ms}, 440 \mathrm{~ms}$, and $169 \mathrm{~ms}$ for IMS, MIP-IMS, and FIM, respectively. It is also observed that when the delay is increased three times, that is, $90 \mathrm{~ms}$, then MDT is $458 \mathrm{~ms}, 596 \mathrm{~ms}$, and $229 \mathrm{~ms}$ for IMS, MIP-IMS, and FIM, respectively. FIM outperforms the others by consuming the lowest MDT.

In Figure 9, for ONP scenario, for a delay of $30 \mathrm{~ms}$, the MDT values are $338 \mathrm{~ms}, 408 \mathrm{~ms}$, and $100 \mathrm{~ms}$ for IMS, MIP-IMS, and FIM, respectively, where FIM outperforms the others by consuming the lowest disruption time.

Figure 10(a) elucidates that, in SP, for a delay $=40 \mathrm{~ms}$, MDT values are $468 \mathrm{~ms}, 578 \mathrm{~ms}$, and $110 \mathrm{~ms}$ for MIP-IMS, IMS, and FIM, respectively. Results prove the dominance of our proposed FIM scheme over preliminaries. It explores that existing schemes endure an abrupt change in MDT values as compared to a steady increase in proposed FIM scheme. In SP case, proposed scheme FIM reduces $71 \%$ compared to IMS scheme and it reduces $83 \%$ MDT compared to MIP-IMS scheme. Figure 10(b) elucidates that, in case of OFP, the MDT values at $40 \mathrm{~ms}$ delay are 468, 609, and 234 for IMS, MIP-IMS, and FIM methods, respectively. It explores that our proposed scheme is faster than the other two schemes. In case of OFP, FIM reduces $50 \%$ as compared to IMS scheme and it reduces $61 \%$ compared to MIP-IMS scheme. Figure 10(c) elucidates that, in case of ONP, for a delay of 40 milliseconds (ms) the MDT values are $468 \mathrm{~ms}, 578 \mathrm{~ms}$, and $140 \mathrm{~ms}$ for MIP-IMS, IMS, and FIM scheme, respectively. Results are evident to elaborate the dominance of our proposed FIM scheme over preliminaries. In ONP case, proposed scheme FIM reduces $71 \%$ MDT compared to IMS scheme and it reduces 77\% MDT compared to MIP-IMS scheme. Figure 10(d) shows the MDT versus delay between UE and new P-CSCF. It elucidates that 


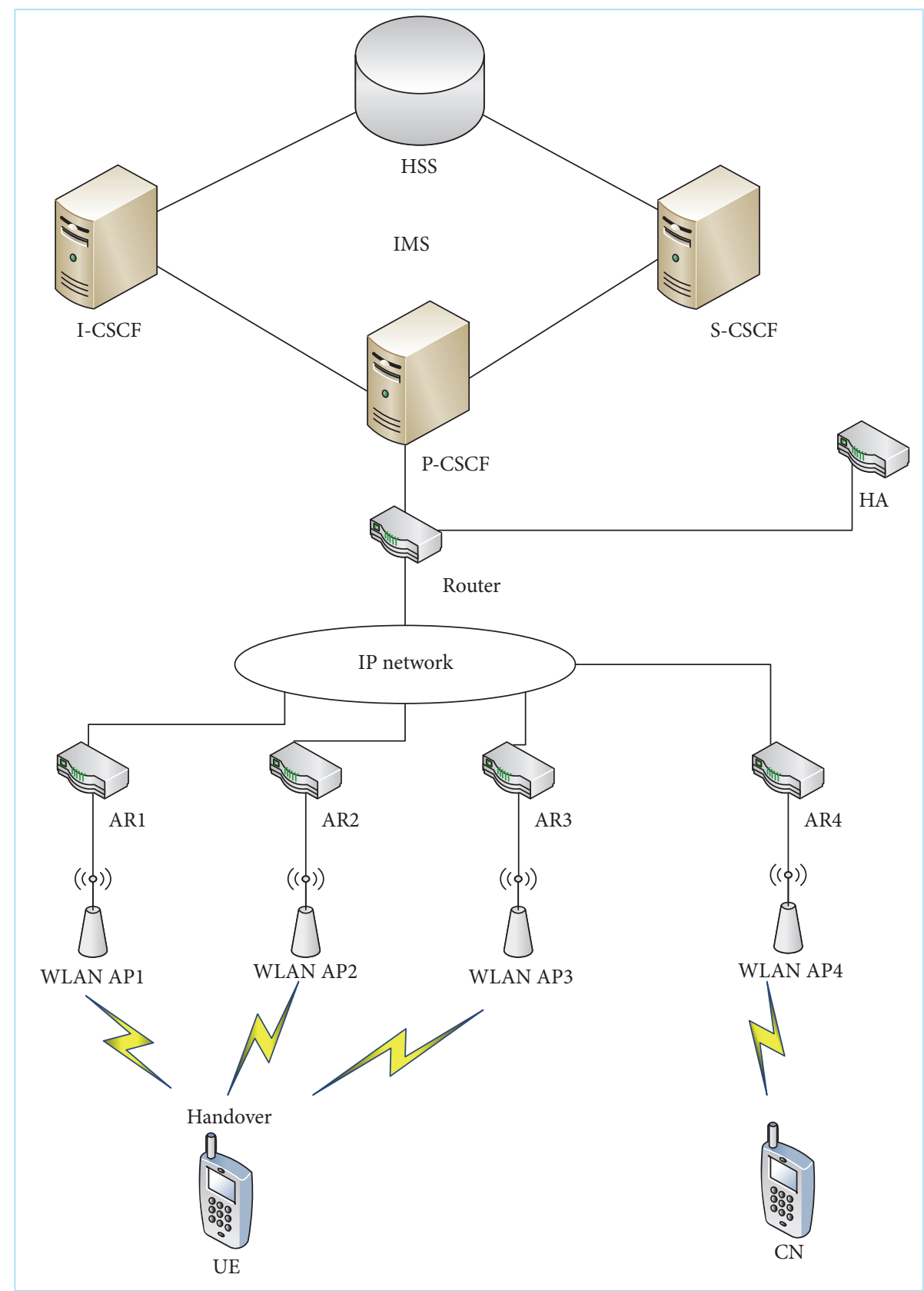

Figure 7: Experimental testbed scenario for FIM scheme.

for a delay of $24 \mathrm{~ms}$ the MDT values are $356 \mathrm{~ms}, 463 \mathrm{~ms}$, and 178 ms for MIP-IMS, IMS, and FIM, respectively. In this case, proposed FIM scheme reduces 50\% MDT compared to IMS scheme and it reduces $61 \%$ MDT compared to MIP-IMS scheme.

Figures 11(a)-11(c) elucidate handover scenario when the number of hops between UE and new AR is varied from 1 to 5 . By presenting values in a sequence of (a) SP, (b) OFP, and (c) ONP for 3-hop scenario, the MDT values are $438 \mathrm{~ms}, 540 \mathrm{~ms}$, and $567 \mathrm{~ms}$ for MIP-IMS and $364 \mathrm{~ms}, 378 \mathrm{~ms}$, and $476 \mathrm{~ms}$ for IMS whereas our FIM scheme consumes only $119 \mathrm{~ms}$, $189 \mathrm{~ms}$, and $155 \mathrm{~ms}$. Figures 11(d)-11(f) elucidate the scenario when the number of hops is varied between UE and $\mathrm{CN}$. By following the same sequence as above, the MDT values are $497 \mathrm{~ms}, 678 \mathrm{~ms}$, and $418 \mathrm{~ms}$ for MIP-IMS and $598 \mathrm{~ms}$, $501 \mathrm{~ms}$, and $544 \mathrm{~ms}$ for IMS, whereas FIM consumes only $140 \mathrm{~ms}, 287 \mathrm{~ms}$, and $121 \mathrm{~ms}$ for SP, OFP, and ONP, respectively. These results are evident to elaborate the dominance of our proposed FIM scheme over preliminaries. Table 2 shows the comparison of the improvement of proposed scheme with IMS and MIP-IMS schemes in terms of percentage for numerical and testbed. It proves that FIM is better in reducing MDT than others when number of hops is varied between UE and new AR and between UE and CN as well. It is observed 


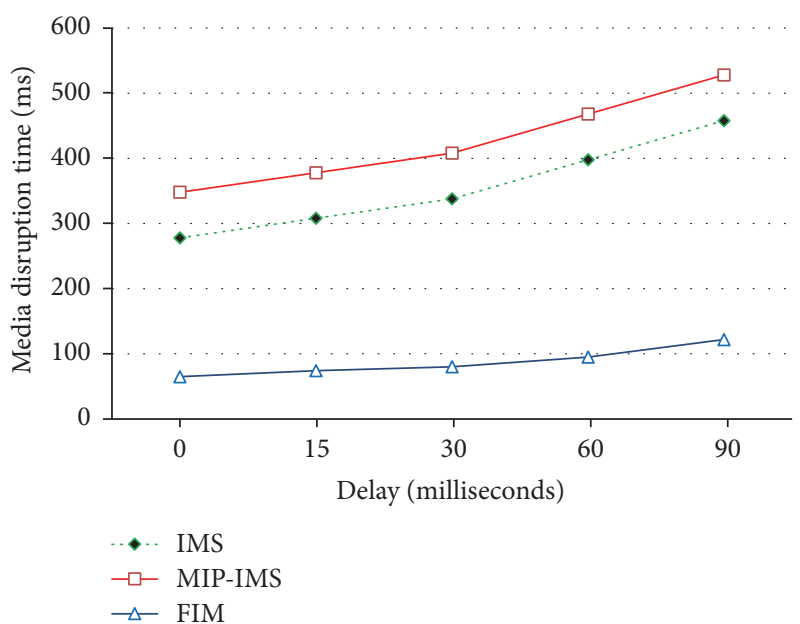

(a)

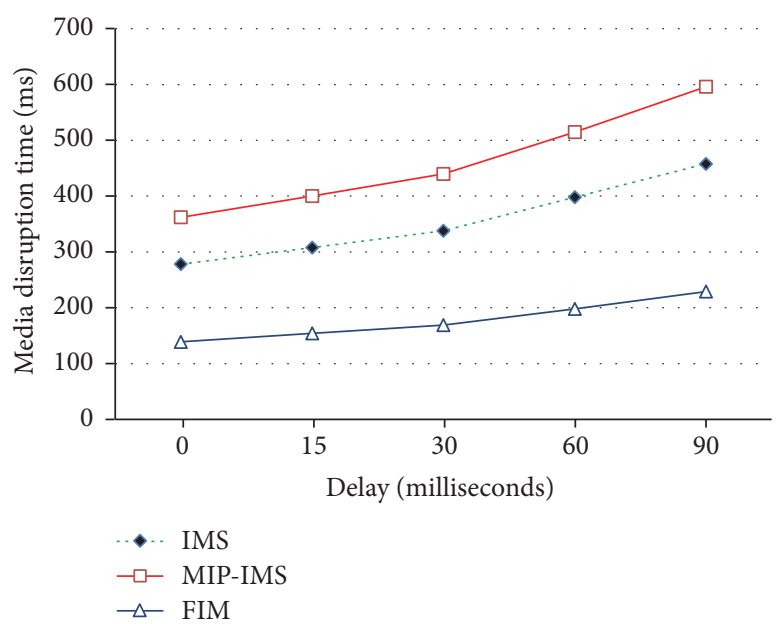

(b)

FIgURE 8: MDT versus delay between UE and AR in (a) for SP and in (b) for OFP.

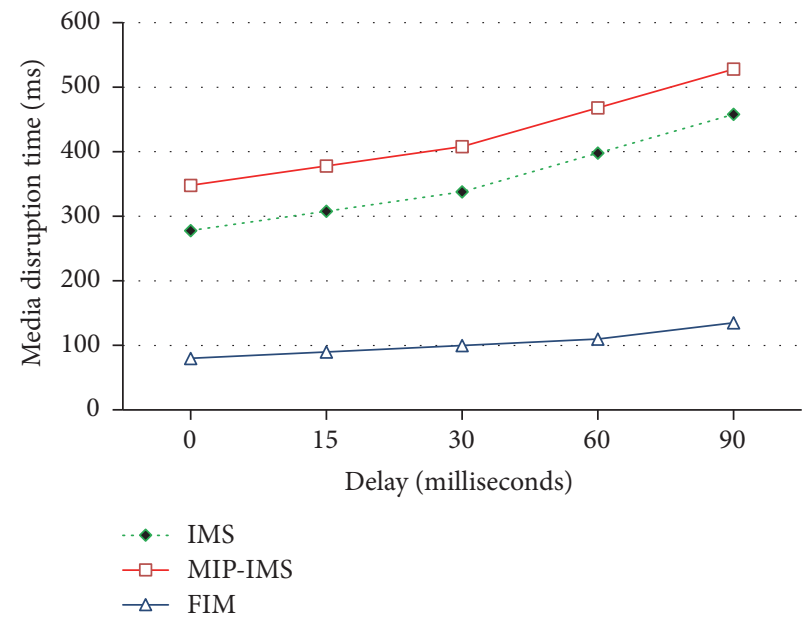

Figure 9: Media disruption time versus delay between UE and ARn in ONP.

that, in case of ONP, MDT is lesser than OFP and it is further reduced in case of SP.

5.2. Packet Loss. We have set $G=50 \mathrm{pkts} / \mathrm{sec}$ as used by [31]. On a constant handover delay, we have measured loss of packets for IMS, MIP-IMS, and FIM. Packet loss is directly proportional to delay of handover [26] as explored in Figure 12(b). During handover, the loss of packets is 44400 bytes, 57600 bytes, and 22200 bytes for IMS, MIP-IMS, and FIM schemes, respectively. FIM reduces the packet loss by $50 \%$ compared to IMS and $61 \%$ compared to MIP-IMS.

5.3. Number of Commands. Our solution reduces the number of $\mathrm{Gm}$ commands between UE and P-CSCF and $M w$ commands between P-CSCF and S-CSCF. Moreover it totally eliminates $C x$ commands between CSCSF servers and HSS. We compare the number of messages of standard IMS handoff scenario and MIP handoff scenarios with FIM handoff approach. Table 2 shows the number of commands for IMS handoff, MIP-IMS handoff, and FIM handoff schemes. Figure 12(a) elucidates that the values of $G m, M w$, and $C x$ are 60, 60 , and 60, respectively, for 10 handovers in case of IMS and MIP-IMS methods. In comparison, values for our proposed scheme are significantly low, that is, 20,20, and 0 , respectively. Our scheme completely removes the need for $C x$ commands.

5.4. SIP Session Delay. To test the delay of our proposed scheme (FIM) for SIP messages, an experiment is run after handover. The delay time of SIP session is captured by using Wireshark. Figure 13 shows the time of SIP session that confirms the reduction of delay.

\section{Conclusion}

NGMN provide a variety of multimedia applications with huge data of live video streaming from cameras or recorded videos for delivering to users. During such data transmissions, mobile UE has to perform handover that can lead to 


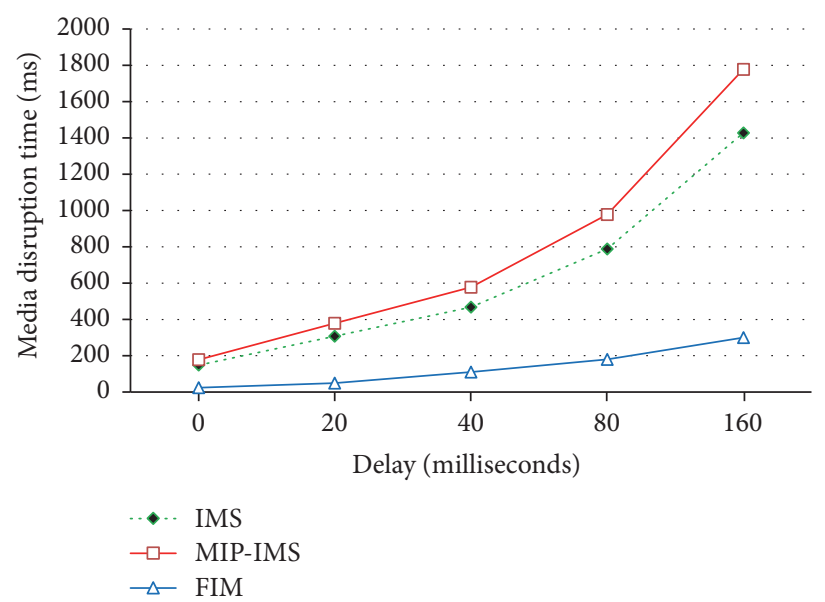

(a)

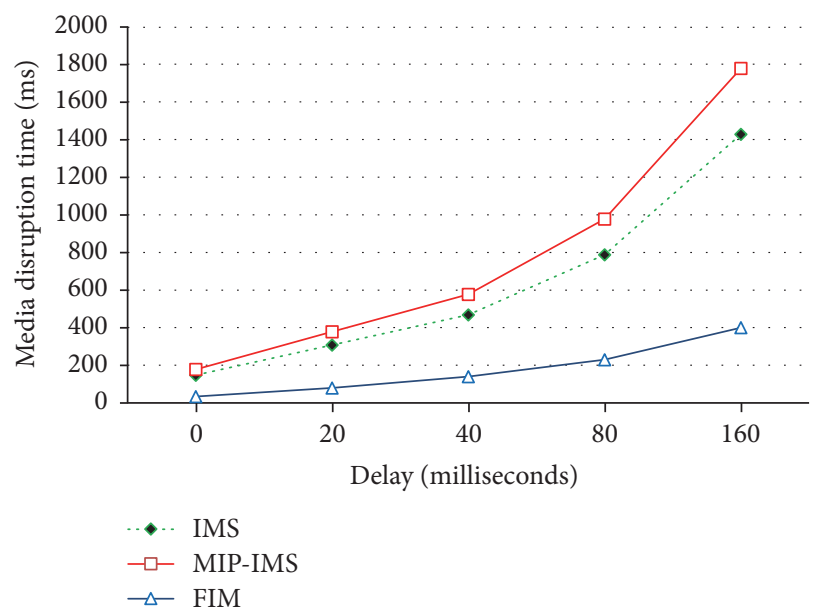

(c)

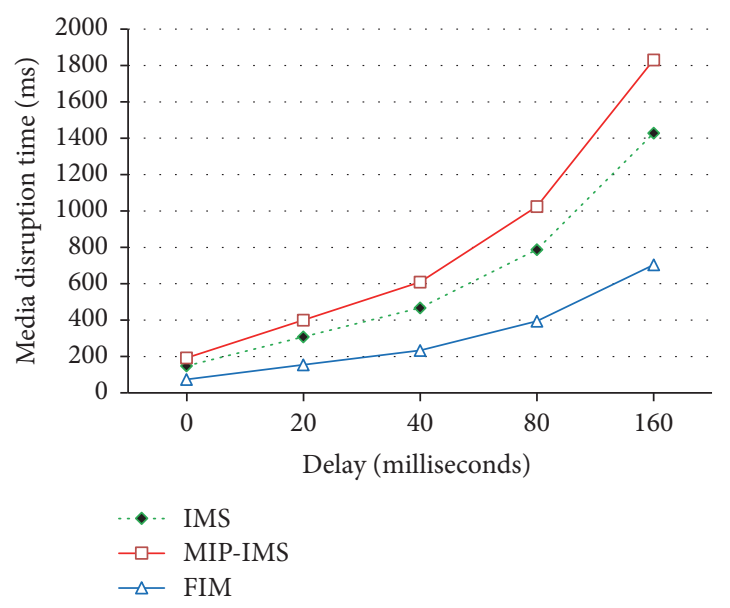

(b)

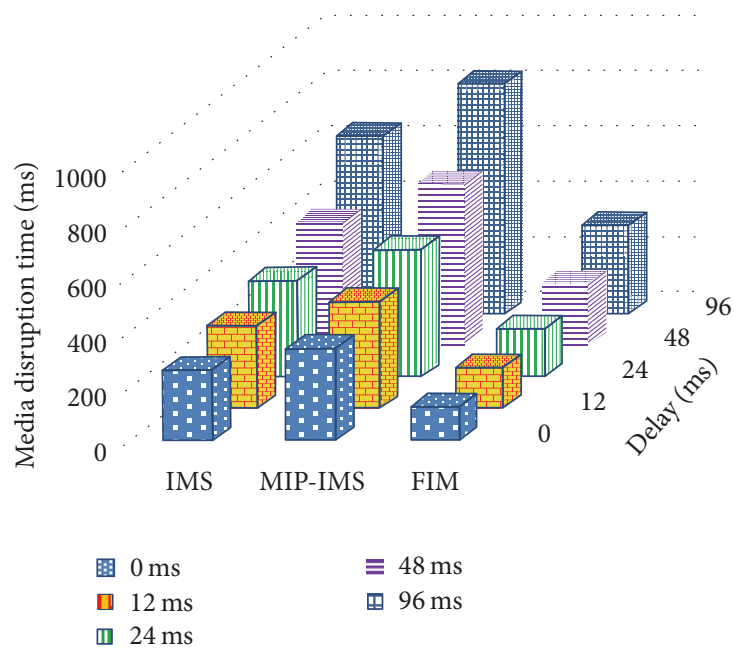

(d)

Figure 10: MDT versus delay between UE and CN for (a) SP, (b) OFP, (c) ONP, and (d) MDT versus delay between UE and new P-CSCF.

TABLE 2: Improvement of FIM scheme over other schemes.

\begin{tabular}{|c|c|c|c|c|c|c|c|}
\hline \multirow{2}{*}{ Number of hops } & \multirow{2}{*}{$\begin{array}{c}\text { Scenarios } \rightarrow \\
\text { Schemes }\end{array}$} & \multicolumn{2}{|c|}{ SP } & \multicolumn{2}{|c|}{ OFP } & \multicolumn{2}{|c|}{ ONP } \\
\hline & & Numerical & Testbed & Numerical & Testbed & Numerical & Testbed \\
\hline \multirow{2}{*}{ Between UE and AR } & IMS & $76 \%$ & $67 \%$ & $50 \%$ & $50 \%$ & $71 \%$ & $65 \%$ \\
\hline & MIP-IMS & $81 \%$ & $72 \%$ & $61 \%$ & $65 \%$ & $77 \%$ & $71 \%$ \\
\hline \multirow{2}{*}{ Between UE and CN } & IMS & $71 \%$ & $71 \%$ & $50 \%$ & $42 \%$ & $71 \%$ & $71 \%$ \\
\hline & MIP-IMS & $83 \%$ & $76 \%$ & $61 \%$ & $57 \%$ & $77 \%$ & $77 \%$ \\
\hline
\end{tabular}

latency and overhead due to disconnection and reregistration. We have presented a session aware registration scheme named FIM for handover scenario where session credentials are transferred without a call session interruption or disconnection. We have developed a testbed using Open IMS to perform experiments by configuring different evaluation parameters for IMS servers and UE. Moreover, algorithms are also proposed for authentication and transfer of IPSec SAs during handover scenario. We have also measured the SIP session delay during handover by using Wireshark. Furthermore the numerical and testbed values are also compared. Results elucidate that FIM reduces MDT by $50 \%$ and $60 \%$ as compared to IMS and MIP-IMS in OFP handover scenario. In case of SP and ONP, our scheme achieves an average of $70 \%$ less MDT consumption. FIM reduces $67 \%$ number of $\mathrm{Gm}$ commands, $67 \%$ of $M w$ commands, and $100 \%$ of 


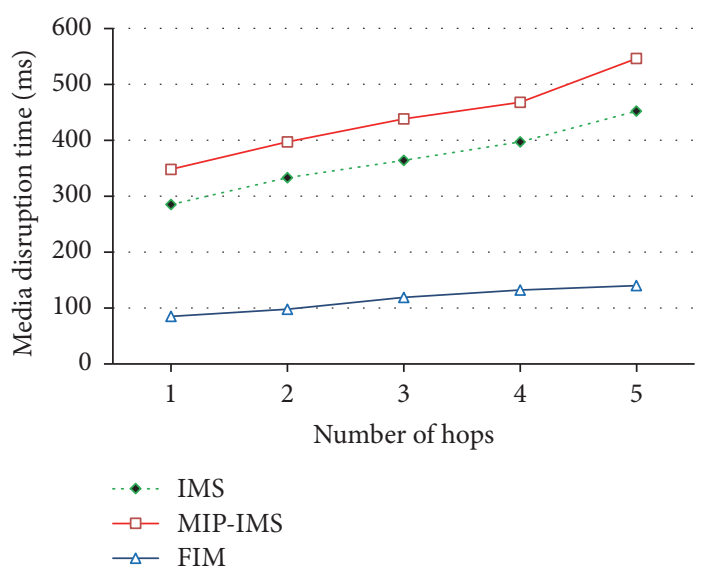

(a)

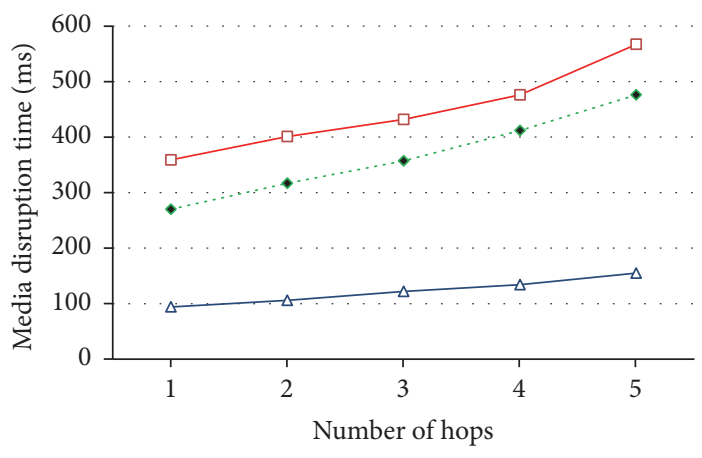

$\begin{array}{ll}-1 & \text { IMS } \\ -\square- & \text { MIP-IMS }\end{array}$

$\neg$ FIM

(c)

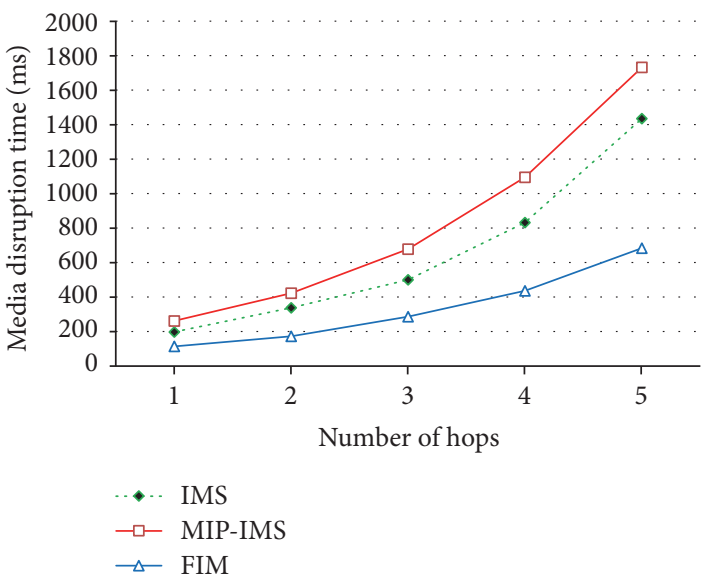

(e)

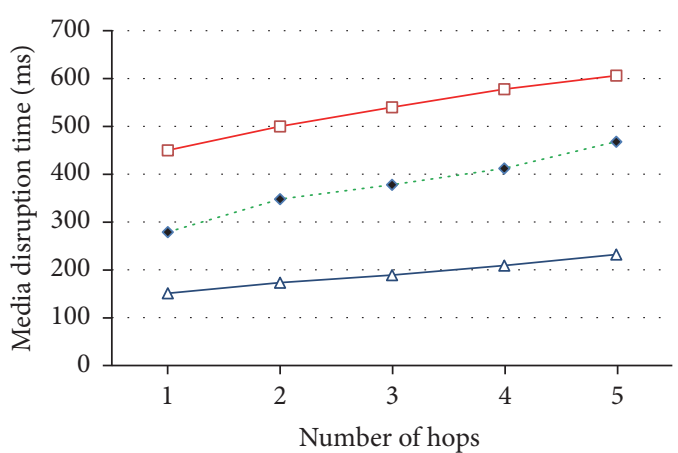

.... IMS

$\rightarrow$ MIP-IMS

$\rightarrow$ FIM

(b)

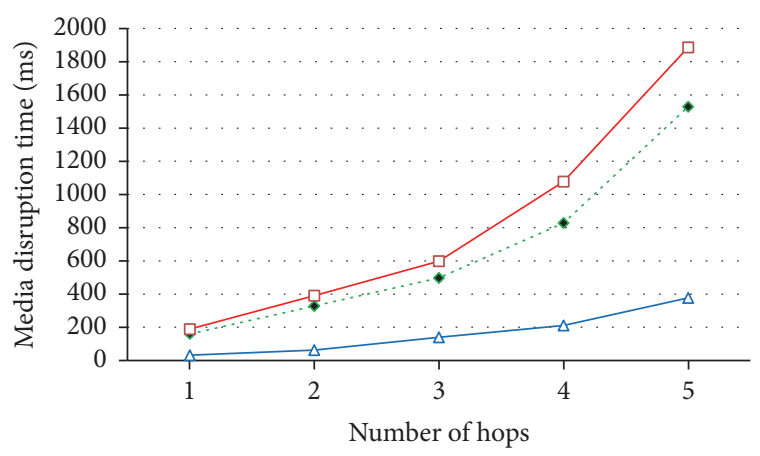

... IMS

$\rightarrow-$ MIP-IMS

$\rightarrow$ FIM

(d)

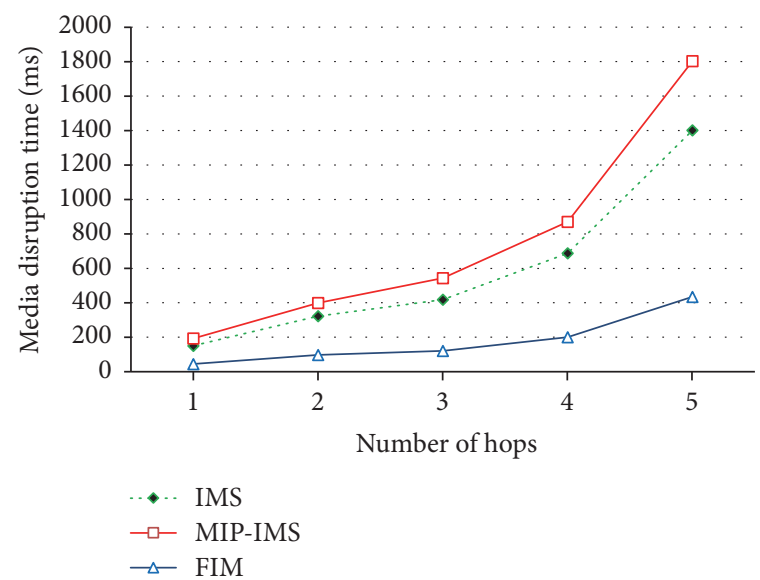

(f)

FIGURE 11: MDT versus number of hops between UE and new AR is presented for (a) SP, (b) OFP, and (c) ONP and between UE and CN is presented in (d) SP, (e) OFP, and (f) ONP scenarios.

$C w$ commands. It also reduces $50 \%$ and $61 \%$ packet loss as compared to IMS and MIP-IMS, respectively. Results prove the dominance of FIM scheme over preliminaries.

\section{Notations}

UE: User equipment
AN: $\quad$ Access network

QoS: Quality of service

MDT: Media disruption time

AR: $\quad$ Access Router

IPSec SAs: IPSec Security Associations

$M w$ : $\quad$ Commands between CSCFs

Gm: $\quad$ Command from UE to IMS 


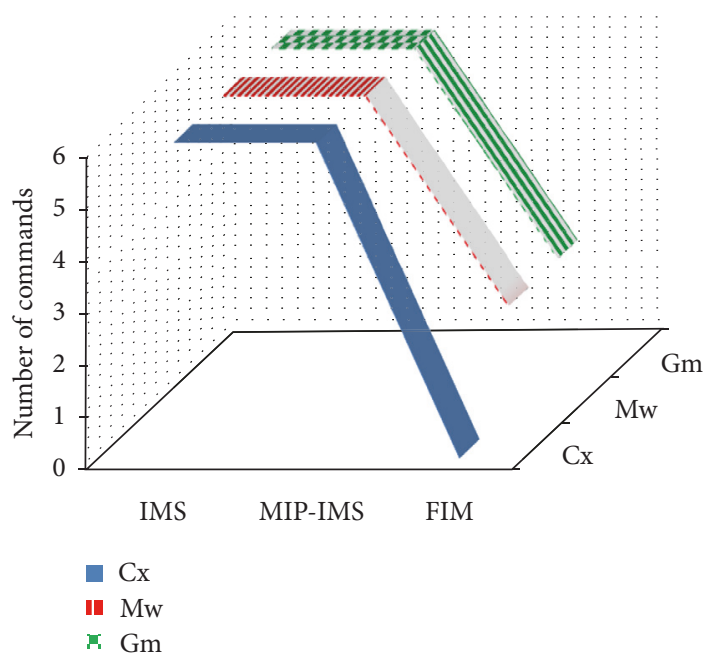

(a)

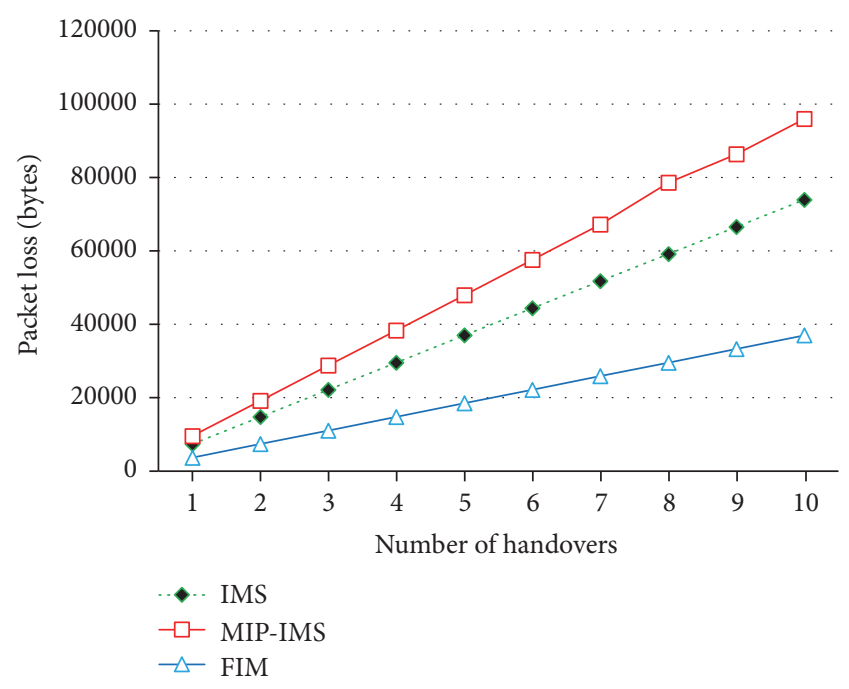

(b)

FIgURE 12: The number of $C x, M w$, and $G m$ commands is presented in (a) and packet loss is presented in (b).

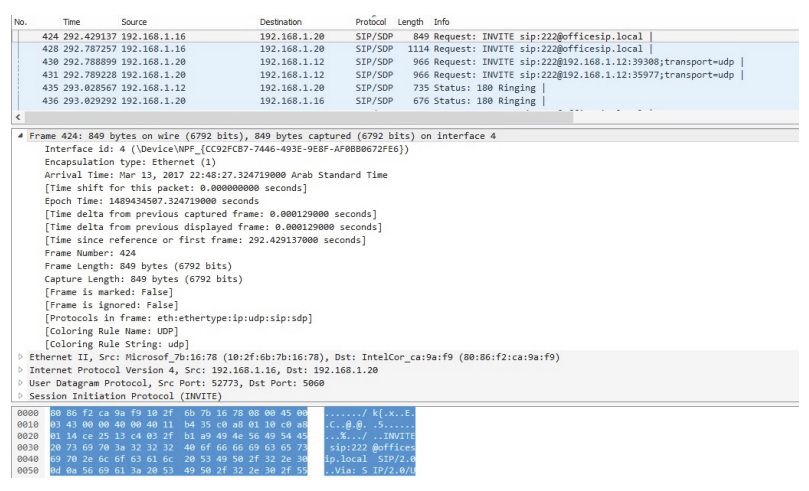

FIGURE 13: Analysis of FIM using Wireshark.

$\begin{array}{ll}\text { Cx: } & \text { Command from CSCFs to HSS } \\ \text { BS: } & \text { Base Station } \\ T: & \text { Time } \\ \text { AP: } & \text { Access Point } \\ \text { HA: } & \text { Home Agent } \\ \text { CoA: } & \text { Care of Address } \\ \text { HoA: } & \text { Home Address } \\ \text { BU: } & \text { Binding Update } \\ \text { BU-Ack: } & \text { BU Acknowledgment } \\ \text { RES: } & \text { Calculated response } \\ \text { IntPr: } & \text { Integrity-Protected } \\ \text { dPCSCF: } & \text { Discovered P-CSCF } \\ \text { TIPSecSA: Old IPSec SA. }\end{array}$

\section{Conflicts of Interest}

The authors declare that there are no conflicts of interest regarding the publication of this paper.

\section{Acknowledgments}

The authors extend their appreciation to the International Scientific Partnership Program ISPP at King Saud University for funding this research work through ISPP no. 0033.

\section{References}

[1] M. Abu-Lebdeh, J. Sahoo, R. Glitho, and C. W. Tchouati, "Cloudifying the 3GPP IP multimedia subsystem for $4 \mathrm{G}$ and beyond: a survey," IEEE Communications Magazine, vol. 54, no. 1, pp. 91-97, 2016.

[2] N. Akkari, "An IMS-based integration architecture for WiMax/ LTE handover," Computer Networks, vol. 57, no. 18, pp. 3790 3798, 2013.

[3] B. Moon, "Fast and secure session mobility in IMS-based vertical handover scenario," International Journal of Multimedia and Ubiquitous Engineering, vol. 9, no. 9, pp. 171-188, 2014.

[4] E. P. Edward, "A novel seamless handover scheme for WiMAX/ LTE heterogeneous networks," Arabian Journal for Science and Engineering, vol. 41, no. 3, pp. 1129-1143, 2016.

[5] E. Prince Edward and V. Sumathy, "Performance analysis of a context aware cross layer scheme for fast handoff in IMS based integrated WiFi-WiMax networks," Pervasive and Mobile Computing, vol. 17, pp. 79-101, 2015.

[6] T. Renier, K. L. Larsen, G. Castro, and H.-P. Schwefel, "Midsession macro-mobility in IMS-based networks," IEEE Vehicular Technology Magazine, vol. 2, no. 1, pp. 20-27, 2007.

[7] K. L. Larsen, E. V. Matthiesen, H.-P. Schwefel, and G. Kuhn, "Optimized macro mobility within the 3GPP IP multimedia subsystem," in Proceedings of the 2nd International Conference on Wireless and Mobile Communications (ICWMC '06), Bucharest, Romania, July 2006.

[8] A. Bagubali, V. Prithiviraj, and P. S. Mallick, "Performance analysis of IMS based LTE and WIMAX integration architectures," Alexandria Engineering Journal, vol. 55, no. 4, pp. 3229-3238, 2016. 
[9] J. T. Renier, F. Hanane, and S. Hans-Peter, "MIP-based enhanced mid-session macro-mobility for IMS-controlled state-full applications," in Proceedings of the 10th International Symposium on Wireless Personal Multimedia Communications, pp. 671-675, Jaipur, India, 2007.

[10] R. Farahbakhsh and N. Movahhedinia, "Seamless handover for IMS over mobile-IPv6 using context transfer," 2012, https:// arxiv.org/abs/1208.1207.

[11] M. Ito, S. Komorita, Y. Kitatsuji, and H. Yokota, "IMS-based fast session handover with available network resources discovery of access networks," Journal of Information Processing, vol. 20, no. 1, pp. 308-318, 2012.

[12] A. Nazari, J. But, P. Branch, and H. Vu, "PRIME: Pre-registration for IMS mobility enhancement," in Proceedings of the 13th IEEE International Conference on Multimedia and Expo (ICME '12), pp. 920-924, Melbourne, VIC, Australia, July 2012.

[13] A. Nazari, P. Branch, J. But, and H. L. Vu, "UPTIME: an IMSbased mobility framework for next generation mobile networks," Wireless Networks, vol. 20, no. 7, pp. 1967-1979, 2014.

[14] J. Rosenberg, S. Henning, C. Gonzalo et al., "SIP: session initiation protocol," RFC 3261, 2002.

[15] D. Johnson, C. Perkins, and J. Arkko, "Mobility support in IPv6," RFC 3775, IETF, 2004.

[16] O. Khattab and O. Alani, "A survey on media independent handover (MIH) and IP multimedia subsystem (IMS) in heterogeneous wireless networks," International Journal of Wireless Information Networks, vol. 20, no. 3, pp. 215-228, 2013.

[17] M. Poikselkä and M. Georg, The IMS: IP Multimedia Concepts and Services, John Wiley \& Sons, Hoboken, NJ, USA, 2013.

[18] R. Koodli, “Mobile IPv6 Fast Handovers," RFC Editor RFC5568, 2009.

[19] N. H. Thanh, L. T. Hang, N. Q. Thu, V. V. Yem, and N. X. Dung, "Multimedia session continuity with context-aware capability in IMS-based network," in Proceedings of the 6th International Symposium on Wireless Communication Systems (ISWCS '09), pp. 383-387, Tuscany, Italy, September 2009.

[20] A. De La Oliva, A. Banchs, I. Soto, T. Melia, and A. Vidal, "An overview of IEEE 802.21: media-independent handover services," IEEE Wireless Communications, vol. 15, no. 4, pp. 96-103, 2008.

[21] S.-R. Yang and W.-T. Chen, "SIP multicast-based mobile quality-of-service support over heterogeneous IP multimedia subsystems," IEEE Transactions on Mobile Computing, vol. 7, no. 11, pp. 1297-1310, 2008.

[22] X. Yang and A. Agarwal, "Multicast mobility in SIP layer," in Proceedings of the IEEE 59th Vehicular Technology Conference, VTC2004-Spring: Towards a Global Wireless World, Milan, Italy, May 2004.

[23] P. Pongpaibool, P. Sotthivirat, S. I. Kitisin, and C. Srisathapornphat, "Fast duplicate address detection for mobile IPv6," in Proceedings of the 15th IEEE International Conference on Networks (ICON '07), pp. 224-229, Adelaide, SA, Australia, aus, November 2007.

[24] X. Yan, Y. A. Şekercioğlu, and S. Narayanan, "A survey of vertical handover decision algorithms in Fourth Generation heterogeneous wireless networks," Computer Networks, vol. 54, no. 11, pp. 1848-1863, 2010.

[25] P. Bellavista, A. Corradi, and L. Foschini, "An IMS vertical handoff solution to dynamically adapt mobile multimedia services," in Proceedings of the 13th IEEE Symposium on Computers and Communications (ISCC '08), pp. 764-771, Marrakech, Morocco, July 2008.
[26] K. S. Munasinghe and A. Jamalipour, "Route optimization for roaming heterogeneous multi-homed mobile networks," in Proceedings of the 4th International Conference on Signal Processing and Communication Systems (ICSPCS '10), Gold Coast, QLD, Australia, December 2010.

[27] G. Camarillo and M.-A. García-Martín, The 3G IP Multimedia Subsystem (IMS): Merging The Internet and The Cellular Worlds, John Wiley \& Sons, Hoboken, NJ, USA, 2007.

[28] T. Magedanz, D. Witaszek, and K. Knuettel, "The IMS playground@ FOKUS-an open testbed for next generation network multimedia services," in Proceedings of the 1st International Conference on Testbeds and Research Infrastructures for the Development of Networks and Communities (Tridentcom '05), pp. 2-11, Trento, Italy, February 2005.

[29] J. Kempf, "Problem description: reasons for performing context transfers between nodes in an IP access network," RFC Editor RFC3374, 2002.

[30] X. Pérez-Costa, M. Torrent-Moreno, and H. Hartenstein, "A performance comparison of Mobile IPv6, Hierarchical Mobile IPv6, fast handovers for Mobile IPv6 and their combination," ACM SIGMOBILE Mobile Computing and Communications Review, vol. 7, no. 4, pp. 5-19, 2003.

[31] L. Cai, Y. Xiao, X. Shen, L. Cai, and J. W. Mark, "VoIP over WLAN: Voice capacity, admission control, QoS, and MAC," International Journal of Communication Systems, vol. 19, no. 4, pp. 491-508, 2006. 


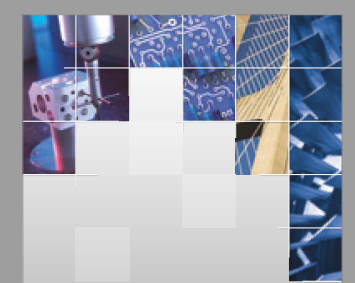

\section{Enfincering}
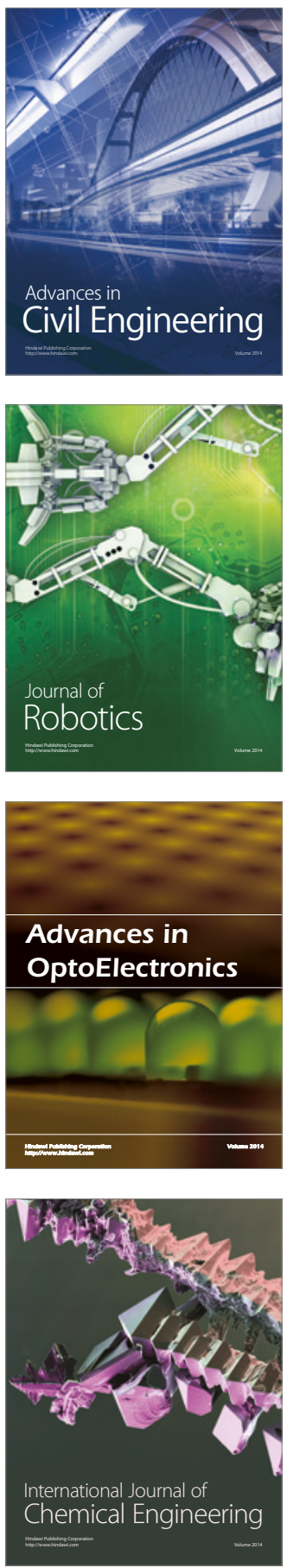

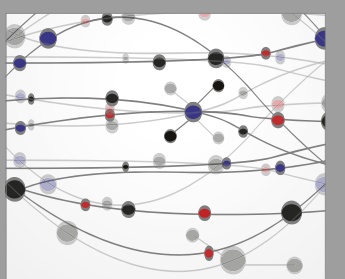

The Scientific World Journal

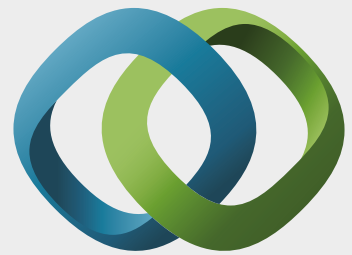

\section{Hindawi}

Submit your manuscripts at

https://www.hindawi.com
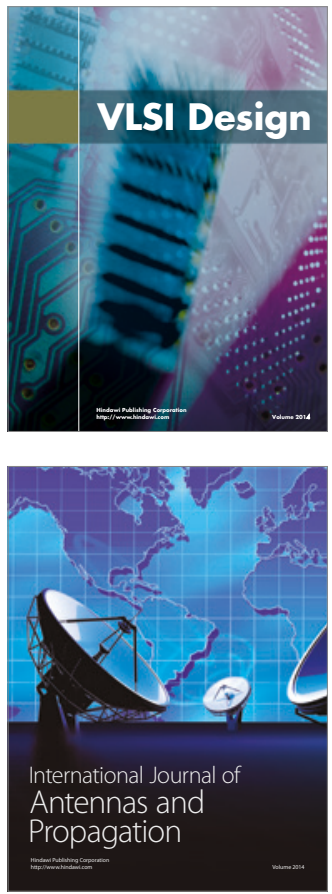

\section{Rotating}

Machinery
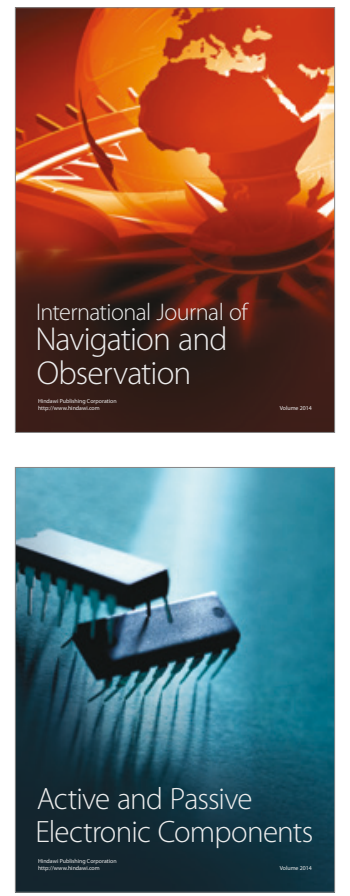
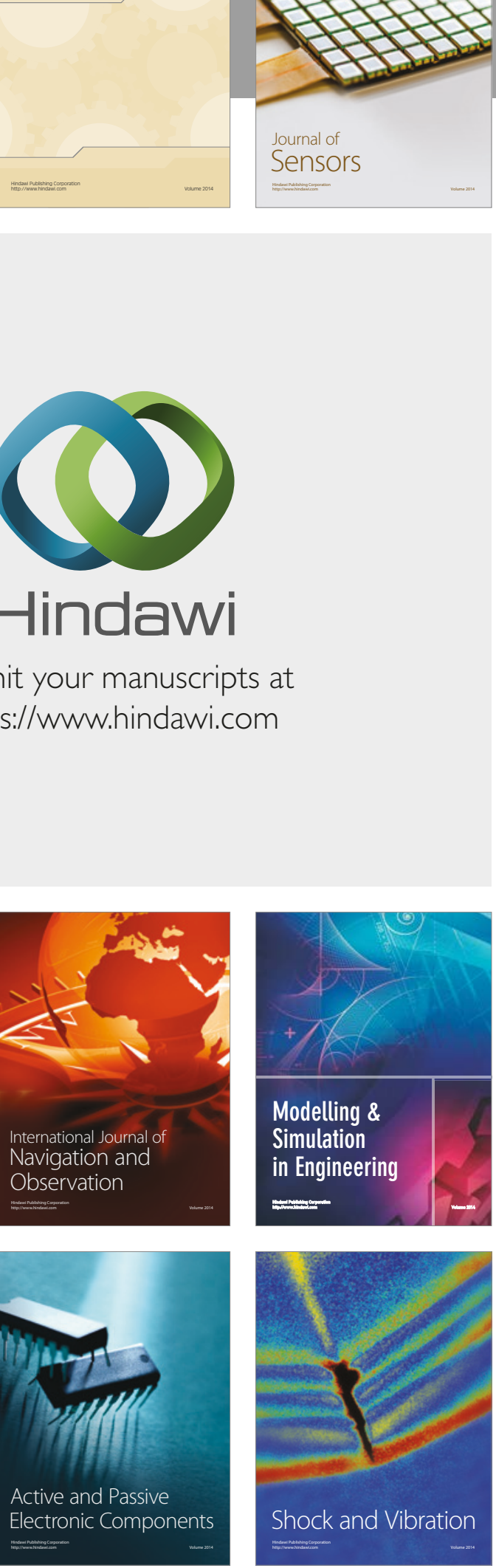
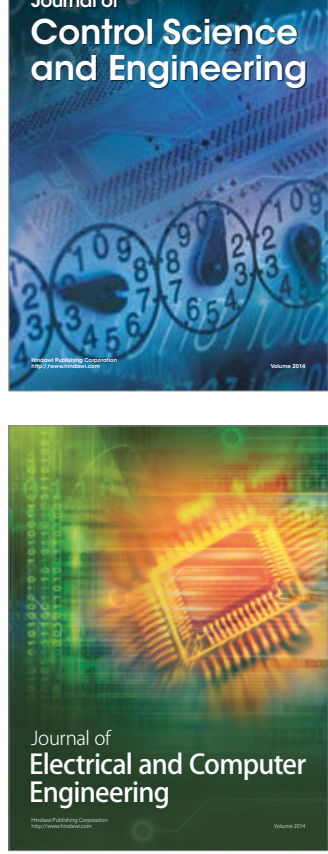

Distributed

Journal of

Control Science

and Engineering
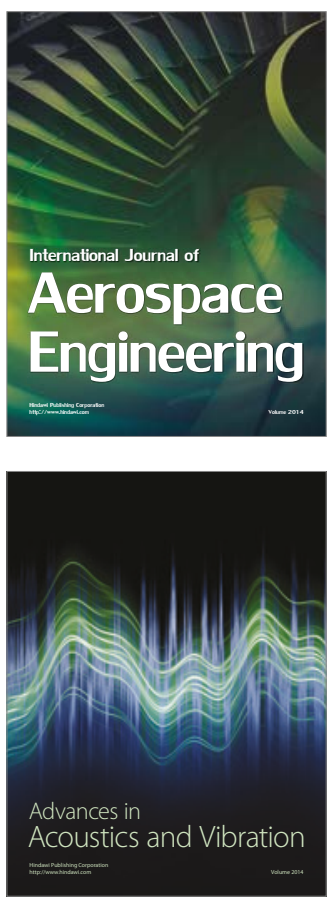

Sensor Networks 